[RADIOCARBON, VOL 28, No. 1, 1986, P 9-68]

\title{
GIF NATURAL RADIOCARBON MEASUREMENTS X
}

GEORGETTE DELIBRIAS, M-T GUILLIER, and JACQUES LABEYRIE

Centre des Faibles Radioactivités, Laboratoire Mixte CNRS-CEA, BP 1

91190 Gif-sur-Yvette, France

The following date list includes archaeologic and geologic samples dated by Gif Radiocarbon Laboratory mostly from 1973 to 1975 . Volcanic samples reported here were dated up to 1981 . Measurements were made in the same manner as previously reported ( $\mathrm{R}, 1972$, v 14, p 280). For undersized samples, a $0.1 \mathrm{~L}, \mathrm{CO}_{2}$ proportional counter was used with 5000-minute standard measurements. Ages listed are conventional ${ }^{14} \mathrm{C}$ ages based on the 5568-year Libby half-life; uncertainties are $1 \sigma$ statistical standard error. Results are based on $95 \%$ of NBS oxalic acid activity. Some dates have been calibrated using the correction curve of Klein et al (1982)*.

\section{ACKNOWLEDGMENTS}

We thank M Kolbach and M Rousseau for sample preparation, as well as J P Garnier and M Jaudon for measurements and maintenance of electronic equipment.

\section{ARCHAEOLOGIC SAMPLES}

France

\section{Palaiseau series, Essonne}

Charcoal from archaeol levels on Plateau de Palaiseau $\left(48^{\circ} 43^{\prime} \mathrm{N}\right.$, $\left.2^{\circ} 14^{\prime} \mathrm{E}\right)$. Coll and subm 1971-1975 by M Cattant, Palaiseau.

Gif-2332. Palaiseau A

$550 \pm 110$

Charcoal from Middle Age occupation.

Gif-3799. Palaiseau B

Charcoal from Merovingian level.

General Comment: dates agree with expected ages.

Gif-3961. Vauhallan, Essonne

$850 \pm 90$

Bones in sarcophagus, from Roman Chapel under church of Vauhallan $\left(48^{\circ} 43^{\prime} \mathrm{N}, 2^{\circ} 14^{\prime} \mathrm{E}\right)$. Coll and subm 1976 by M Cattant.

\section{Bois de Saponay series, Aisne}

Charcoal samples from level overlying Tardenoisian level of Bois de Saponay site $\left(49^{\circ} 12^{\prime} \mathrm{N}, 3^{\circ} 32^{\prime} \mathrm{E}\right)$. Coll and subm 1972 by J Hinout, Chateau-Thierry, Aisne.

* Calibrated dates will now be reported as "cal BP" according to the consensus of ${ }^{14} \mathrm{C}$ daters at the 12th International Radiocarbon Conference. 
Gif-2450. Bois de Saponay, P 19-145

Gif-2451. Bois de Saponay, N 20-150

General Comment: younger than Tardenoisian age, as expected from strat position of charcoal.

Gif-2403. Crèvecoeur-en-Auge, Calvados

Wood from ancient drawbridge of castle of Crèvecoeur-en-Auge $\left(49^{\circ}\right.$ $07^{\prime} \mathrm{N}, 0^{\circ} 01^{\prime} \mathrm{E}$ ). Coll and subm 1971 by R Jost, Mus Schlumberger, Crèvecoeur-en-Auge. Comment: date is consistent with hist data.

Gif-2307. Le Plessis-Grimoult, Calvados

$1340 \pm 70$

Charcoal at $3.5 \mathrm{~m}$ depth, under circular rampart around medieval bldg, Le Plessis-Grimoult $\left(48^{\circ} 57^{\prime} \mathrm{N}, 0^{\circ} 36^{\prime} \mathrm{W}\right)$. Coll 1970 by M Rio and subm 1971 by M de Bouard, Centre Recherche Archaeol Médiévales, Caen. Comment: dates limit for bldg.

\section{Montgasteau series, Saint Denis-les-Ponts, Eure et Loir}

Charcoal from Neolithic mound inside rampart of promontory camp of Mongasteau ( $\left.48^{\circ} 04^{\prime} \mathrm{N}, 1^{\circ} 17^{\prime} \mathrm{E}\right)$. Coll by M Haricot and subm 1973 by J Allain, Dir Antiquités Préhist, Bourges.

Gif-2772. Montgasteau, SP, 72-06 $4790 \pm 130$ Depth $3.4 \mathrm{~m}$.

Gif-2773. Montgasteau, SP, 72-07 $4860 \pm 130$ Depth $2.9 \mathrm{~m}$.

Gif-2774. Montgasteau, SP, 72-08 $4550 \pm 130$ Depth $1.7 \mathrm{~m}$.

General Comment: dates this type of military structure.

Gif-2455. Chaudron, Maine et Loire

$1200 \pm 90$

Charcoal from artificial souterrain of $\mathrm{L}$ a Ravaillière, Chaudron $\left(47^{\circ}\right.$ $17^{\prime} \mathrm{N}, 0^{\circ} 59^{\prime} \mathrm{W}$ ). Coll and subm 1972 by J L'Helgouach, Dir Antiquités Préhist, Nantes.

Gif-2290. Lavardin, Bellevue, Sarthe

$1270 \pm 100$

Ashes in hearth, from ancient furnace from iron foundry settlement, Lavardin $\left(48^{\circ} 05^{\prime} \mathrm{N}, 0^{\circ} 05^{\prime} \mathrm{E}\right)$. Coll and subm 1971 by A Pioger, Le Mans, Sarthe. Comment: iron mining in Sarthe is dated to 8th century.

Gif-2745. Saint-Fort, Mayenne

$1380 \pm 90$

Wood in filling of ancient gold mine, Saint-Fort $\left(47^{\circ} 49^{\prime} \mathrm{N}, 0^{\circ} 42^{\prime} \mathrm{W}\right)$. Subm 1973 by J Guignes, Bur Recherche Géol et Min, Rennes. Comment: dates medieval gold mining in Mayenne. 
Wood from dug-out canoe found in silty sediments of La Taute $\mathrm{R}$, Rauville-la-Place $\left(49^{\circ} 22^{\prime} \mathrm{N}, 1^{\circ} 30^{\prime} \mathrm{W}\right)$. Coll and subm 1972 by $\mathrm{M}$ de Bouard.

Gif-3206. Plonevez-Porzay, Kervel, Finistère

$1950 \pm 90$

Charcoal from ancient salt industry site Plovenez-Porzay $\left(48^{\circ} 04^{\prime} \mathrm{N}, 4^{\circ}\right.$ $05^{\prime}$ W). Coll and subm 1973 by R Sanquer, Lab Archaeol Brest. Comment: cal 175 BC-AD 235. Date agrees with expected date, AD 275, from ceramics.

\section{Pluguffan, Kerbernard series, Finistère}

Charcoal from Kerbernard barrows, Pluguffan $\left(47^{\circ} 57^{\prime} \mathrm{N}, 4^{\circ} 12^{\prime} \mathrm{W}\right)$. Coll 1973 and subm 1974 by J Briard, Fac Sci, Rennes.

\section{Gif-3202. Kerbernard 2}

Classic Breton Bronze age tombstone. Comment: date agrees with archaeol data.

Gif-3203. Kerbernard 3

$1620 \pm 90$

Charcoal from chest, assoc with Chalcolithic industry. Comment: date is too young, indicating site disturbance.

Gif-3201. Priziac, Morbihan

$3930 \pm 110$

Charcoal from Early Bronze age barrow, at Priziac $\left(48^{\circ} 04^{\prime} \mathrm{N}, 3^{\circ}\right.$ 06' W). Coll 1973 and subm 1974 by J Briard.

\section{Laniscat series, Côtes du Nord}

Charcoal from Megalithic tomb, Laniscat $\left(48^{\circ} 13^{\prime} \mathrm{N}, 3^{\circ} 08^{\prime} \mathrm{W}\right)$. Coll 1973 and subm 1975 by C T Le Roux, Fac Sci, Rennes.

Gif-3200. Laniscat, 3 T 4

$$
2860 \pm 100
$$

Charcoal under flagstone.

Gif-3099. Laniscat, 2

Charcoal from entrance of Megalithic monument.

General Comment: Gif-3099 gives good date for closing of tomb; Gif-3200 indicates later re-use.

Gif-3204. Pedernec, Trezean, Côtes du Nord

Charcoal in Chamber A from Iron Age souterrain, Pedernec, Trezean $\left(48^{\circ} 34^{\prime} \mathrm{N}, 3^{\circ} 18^{\prime} \mathrm{W}\right)$. Coll and subm 1973 by P R Giot, Fac Sci, Rennes. Comment: fits archaeol evidence.

Gif-3205. Kermoysan, Quimper, Finistère

Charcoal from hearth, at base of furnace, Kermoysan $\left(48^{\circ} 00^{\prime} \mathrm{N}, 4^{\circ} 06^{\prime}\right.$ W). Coll 1972 and subm 1973 by R Sanquer. Comment: cal 400 BC-AD 40, 
agrees with expected date (AD 10-25) for first Roman settlement of Quimper.

\section{Colpo series, Morbihan}

Samples from Cairn II, part of littoral Megalithic site of Larcuste, Colpo $\left(47^{\circ} 51^{\prime} \mathrm{N}, 2^{\circ} 47^{\prime} \mathrm{W}\right)$. Coll and subm 1972-1973 by J L'Helgouach.

\section{Gif-2828. Colpo 5}

Charcoal from $E$ side, $S$ entrance of cairn.

Gif-2453. Colpo 1

$3980 \pm 110$

Charcoal from E side, inside cairn.

\section{Gif-2827. Colpo 4}

$4060 \pm 120$

Charcoal from $\mathrm{N}$ side, inside cairn.

Gif-2454. Colpo $\mathbf{f} 2$

$4610 \pm 110$

Charcoal from same strat position as Gif-2453, but inside cairn.

\section{Gif-2826. Colpo 3}

$5490 \pm 120$

Charcoal from passage-grave a-b, assoc with Neolithic ceramics.

General Comment: Gif-2826 is most interesting date, from construction level of passage-grave.

\section{Ancenis series, Loire Atlantique}

Samples from ancient dike of Loire R, Ancenis $\left(47^{\circ} 23^{\prime} \mathrm{N}, 1^{\circ} 10^{\prime} \mathrm{W}\right)$. Coll and subm 1972 by J L'Helgouach.

\section{Gif-2456. Ancenis I}

Wood from pile.

$$
970 \pm 90
$$

\section{Gif-2457. Ancenis II}

$$
1010 \pm 90
$$

Wood from another pile. Comment: dates bldg of dikes of Loire R.

\section{Gif-2901. Saint-Michel-Chef-Chef, Loire Atlantique}

$5200 \pm 90$

Charcoal from Paleolithic site in Loire estuary, Saint-Michel-ChefChef $\left(47^{\circ} 11^{\prime} \mathrm{N}, 2^{\circ} 09^{\prime} \mathrm{W}\right)$. Coll by M Allard and subm 1973 by J L'Helgouach. Comment: dated charcoal was not in situ, probably from upper archaeol levels.

\section{Gif-2347. Courcoury, Charente Maritime}

Ox bones found in barrow of Courcoury $\left(45^{\circ} 44^{\prime} \mathrm{N}, 0^{\circ} 38^{\prime} \mathrm{W}\right), 5 \mathrm{~m}$ from top. Subm 1971 by M Hours, Dir Mus France, Paris. Comment: dates this large barrow, $12 \mathrm{~m}$ high, $70 \mathrm{~m}$ diam, for which no archaeol data are available. 


\section{Grotte du Queroy series, Charente Maritime}

Samples from protohist levels of Grotte du Queroy $\left(45^{\circ} 39^{\prime} \mathrm{N}, 0^{\circ} 19^{\prime}\right.$ E). Coll and subm 1973-1978 by J Gomez, Dir Antiquités Préhist, Poitou, Charentes.

Gif-2742. Grotte du Queroy, Level 2

$$
2400 \pm 110
$$

Charcoal in hearth of La Tène age.

Gif-2741. Grotte du Queroy, Level 3a, Pit 5

$1820 \pm 100$

Charcoal, end of First Iron age.

Gif-3283. Grotte du Queroy, Level 3a

$2070 \pm 90$

Charcoal, end of First Iron age.

Gif-4677. Grotte du Queroy, Level 4

$$
2610 \pm 90
$$

Charcoal from Level 4 of pit in entrance, end of First Iron age.

Grotte-3284. Grotte du Queroy, Hearth 1

$2670 \pm 100$

Charcoal from Hearth 1 in habitation soil with Late Bronze age-Hallstatt B3 industry (Venat Group), lying on Level 5.

Gif-3775. Grotte du Queroy, Hearth 2

Charcoal in Hearth 2 from same level as Hearth 1. Comment: with Gif3284 , dates precisely first appearance of iron objects at site.

Gif-2740. Grotte du Queroy, Level 5

$2820 \pm 110$

Scattered charcoal from level with typical "Venat Group" industry.

Gif-4678. Grotte du Queroy, B

$2940 \pm 100$

Charcoal, Late Bronze III, from another part of Cave.

Gif-2739. Grotte du Queroy, Level 7

$3040 \pm 110$

Charcoal, from Middle Bronze to early Late Bronze age.

Gif-4127. Grotte du Queroy, Level 8

Charcoal from carbonized post, Middle Bronze age II-III.

Gif-3285. Grotte du Queroy, Level 9

$4260 \pm 110$

Charcoal in ossuary in reworked level of Artenac level; assoc with copper objects.

General Comment: except for unexplained dates of Gif-2741 and -3283, this series provides good chronology for Bronze and Iron ages in $\mathrm{W}$ France.

Gif-2743. Pierre-Dure, Voeuil-et-Giget, Charente Maritime $4150 \pm 130$

Charcoal from Neolithic camp of Pierre-Dure $\left(45^{\circ} 36^{\prime} \mathrm{N}, 0^{\circ} 10^{\prime} \mathrm{E}\right)$. Coll 1969 and subm 1973 by J Gomez. Comment: fits very well for this site of Artenac culture (Gomez, 1975). 


\section{Gencay series, Vienne}

Samples from anterior level to present ruins of ancient castle of Gencay $\left(46^{\circ} 25^{\prime} \mathrm{N}, 0^{\circ} 23^{\prime} \mathrm{E}\right)$. Coll and subm 1972 by A Vignaud, Dir Antiquités Préhist, Poitiers.

Gif-2645.

$810 \pm 90$

Burned corn.

Gif-2646.

$1050 \pm 90$

Charcoal.

General Comment: dates are coherent with hist data, castle was built during 13 th century.

\section{Grotte du Bois-Ragot series, Gouex, Vienne}

Samples from Late Magdalenian levels of Grotte du Bois-Ragot, Gouex ( $45^{\circ} 23^{\prime} \mathrm{N}, 1^{\circ} 38^{\prime} \mathrm{W}$ ). Coll 1971 and subm 1972 by A Chollet, Chatellerault, Vienne.

Gif-3580. Grotte du Bois-Ragot, Level VI

$10,990 \pm 160$

Charcoal.

Gif-2537. Grotte du Bois-Ragot, Level V

$11,030 \pm 140$

Carbonaceous ashes.

Gif-3579. Grotte du Bois-Ragot, Level VI

Carbonaceous earth.

General Comment: dates agree with reindeer bone industry found in these levels (Chollet, Reigner, \& Boutin, 1974). Azilian level of site was dated, $8800 \pm 220$, Gif-1588 (R, 1974, v 16, p 25).

Gif-2419. Thiers, Puy de Dôme

$1770 \pm 100$

Wood from ancient water pipe, near Thiers $\left(45^{\circ} 51^{\prime} \mathrm{N}, 3^{\circ} 33^{\prime} \mathrm{E}\right)$. Coll 1971 by J Tournet and subm 1972 by G Camus, Univ Clermont-Ferrand. Comment: date agrees with Roman age attributed to aqueduct.

\section{Grotte du Rond du Barry series, Polignac, Haute Loire}

Samples from Grotte du Rond du Barry, Polignac ( $\left.45^{\circ} 04^{\prime} \mathrm{N}, 3^{\circ} 52^{\prime} \mathrm{E}\right)$. Coll and subm 1972-73-75 by R de Bayle des Hermens, Mus l'Homme, Paris (de Bayle des Hermens, 1977).

Gif-3738. Grotte du Rond du Barry, 75-1

$860 \pm 60$

Charcoal from Middle age level.

Gif-2671. Grotte du Rond du Barry, Level D, 72-1 $\quad 12,380 \pm 280$

Carbonaceous earth from hearth in Upper Magdalenian level.

Gif-2672. Grotte du Rond du Barry, Level E, 72-2 $\quad \mathbf{1 5 , 4 0 0} \pm 400$

Burned bones from Hearth 2 in Upper Magdalenian level. 
Gif-3492. Grotte du Rond du Barry, Level E, 73-3 12,800 \pm 170

Burned bones from Hearth 4 in Upper Magdalenian level.

Gif-3038. Grotte du Rond du Barry, Level F, 73-5 17,100 \pm 450

Splintered bones from Upper Magdalenian level.

General Comment (RBH): all dates agree well with archaeol evidence, but Gif3492 , from same level as Hearth 2, is too young.

Gif-2312. “Couvent des Fieux," Miers, Lot

$1150 \pm 90$

Charcoal from dolmen of "Couvent des Fieux" (44 $\left.51^{\prime} \mathrm{N}, 1^{\circ} 41^{\prime} \mathrm{E}\right)$. Coll and subm 1971 by M Carrière, Vayrac, Lot. Comment: date is much younger than expected.

\section{Le Frau series, Cazals, Tarn et Garonne}

Samples from First Iron age barrow, Le Frau, near Cazals $\left(44^{\circ} 07^{\prime} \mathrm{N}\right.$, $1^{\circ} 40^{\prime} \mathrm{E}$ ). Coll and subm 1971 by B Pajot, Mus Hist Nat, Toulouse.

Gif-2461. Le Frau, Barrow 1

$2140 \pm 100$

Charcoal from Barrow 1, Sq H 8.

Gif-2462. Le Frau, Barrow 2

$1170 \pm 90$ site.

Charcoal from Barrow 2, Sq D 7. Comment: probably dates re-use of

\section{Marquay series, Grotte des Partisans, Dordogne}

Samples from burial cave of Middle Bronze age, Marquay $\left(44^{\circ} 55^{\prime} \mathrm{N}\right.$, $\left.1^{\circ} 07^{\prime} \mathrm{E}\right)$. Coll and subm 1975 by J Roussot-Larroque, CNRS, Bordeaux.

Gif-3595. Marquay, 1

$2540 \pm 120$

Charcoal in mass of fallen earth. Comment: younger than expected; contamination with younger charcoal is suspected.

Gif-3793. Marquay, 2

$3520 \pm 110$

Charcoal from Level 1a-1b. Comment: agrees with archaeol estimate.

Gif-3596. Grotte de Leygonie, Neuvic-sur-l'Isle, Dordogne $3660 \pm 150$

Charcoal from sepulchral cave of Leygonie $\left(45^{\circ} 05^{\prime} \mathrm{N}, 0^{\circ} 30^{\prime} \mathrm{E}\right)$. Coll and subm 1975 by J Roussot-Larroque. Comment: probably dates last occupation of cave, Late Chalcolithic period; undersized sample.

Gif-2384. Grotte d'Eybral, Coux-et-Bigaroque, Dordogne $4140 \pm 140$

Charcoal from burial cave with remains of 60 skeletons, Coux-et-Bigaroque $\left(44^{\circ} 50^{\prime} \mathrm{N}, 0^{\circ} 59^{\prime} \mathrm{W}\right)$. Coll and subm 1971 by $\mathrm{J}$ Roussot-Larroque. Comment: agrees with assoc Late Neolithic industry.

\section{Fontaine de la Demoiselle series, Saint-Léon-sur-l'Isle, Dordogne}

Samples from Neolithic site, La Fontaine de La Demoiselle $\left(45^{\circ} 06^{\prime} \mathrm{N}\right.$, $0^{\circ} 30^{\prime} \mathrm{E}$ ). Coll and subm 1972 by $\mathrm{J}$ Roussot-Larroque. 
Gif-2617. Fontaine de La Demoiselle, 1

Charcoal from Layer B1.

Gif-2618. Fontaine de La Demoiselle, 3

Charcoal from Layer A2 underlying B1.

General Comment: dates agree with Neolithic period with Artenac assoc; dates confirm previous dates for site (R, 1974, v 16, p 25).

\section{Gif-2570. “Pont d'Ambon”, Bourdeilles, Dordogne}

$9830 \pm 180$

Bones from Azilian site, Pont d'Ambon, Bourdeilles (49 $10^{\prime} \mathrm{N}, 0^{\circ} 35^{\prime}$ E). Coll and subm 1972 by G Celerier, Univ Bordeaux.

\section{La Ferrassie series, Dordogne}

La Ferrassie $\left(44^{\circ} 56^{\prime} \mathrm{N}, 1^{\circ} 02^{\prime} \mathrm{E}\right)$ is important archaeol site, with accumulation of deposit 7 to $8 \mathrm{~m}$ thick, containing abundant lithics from Mousterian to Perigordian periods. Site is well known for discovery of 6 Neandertal skeletons in lower level during first excavation period, 1896-1929. H Delporte and A Tuffreau resumed excavations in 1968 (Delporte, 1985). Charcoal was found in only one level. Dating was attempted on abundant faunal bones found in all levels. Bone collagen was extracted according to Longin (1971). Samples come from 2 perpendicular secs: sagittal and frontal; subm 1972-74 by H Delporte, Mus Saint-Germain-en-Laye.

Sagittal section

Gif-2696. La Ferrassie, D 2

$$
24,000 \pm 550
$$

Bones from Level D 2, Perigordian V industry assoc.

Gif-2698. La Ferrassie, D 2

$24,600 \pm 550$

Bones from Level D 2, Perigordian V industry assoc.

Gif-2699. La Ferrassie, D 2

$22,500 \pm 500$

Bones from Level D 2, Perigordian V industry assoc.

Gif-2701. La Ferrassie, EL s

$23,600 \pm 550$

Bones from Level EL s, Aurignacian IV industry assoc.

Gif-4263. La Ferrassie, E 1 s A

$11,150 \pm 120$

Bones from Level E $1 \mathrm{~s} \mathrm{~A}$, Aurignacian IV industry assoc. Comment: date is aberrant.

Gif-4264. La Ferrassie, EL s B

$23,700 \pm 250$

Bones from Level EL s B, Aurignacian IV industry assoc.

Gif-4266. La Ferrassie, G O

$26,100 \pm 210$

Bones from Level G O, Aurignacian III industry assoc.

Gif-4267. La Ferrassie, G 1

$21,100 \pm 170$

Bones from Level G 1, Aurignacian III industry assoc. 
Gif-4268. La Ferrassie, G 1 s b/c

Bones from Level G $1 \mathrm{~s} \mathrm{~b} / \mathrm{c}$, Aurignacian III industry assoc.

Gif-4269. La Ferrassie, G 1 s c

$23,700 \pm 240$

Bones from level G $1 \mathrm{~s} \mathrm{c}$, Aurignacian III industry assoc.

Gif-4270. La Ferrassie, G 1 s c/d

$23,000 \pm 240$

Bones from Level G $1 \mathrm{~s} \mathrm{c} / \mathrm{d}$, Aurignacian III industry assoc.

Frontal section

Gif-2700. La Ferrassie, E 1

$22,500 \pm 500$

Bones from Level E 1, Perigordian V industry assoc.

Gif-4265. La Ferrassie, $F$

$22,200 \pm 650$

Bones from Level F, Aurignacian III-IV industry assoc.

Gif-4271. La Ferrassie, I 1

$28,700 \pm 250$

Bones from Level I 1, Aurignacian III industry assoc.

Gif-4272. La Ferrassie, I 2-I

$25,500 \pm 250$

Bones from Level I 2, Aurignacian III industry assoc.

Gif-4272bis. La Ferrassie, I 2-11

$6300 \pm 100$

Mineral fraction of bones from Level I 2. Comment: date shows contamination.

Gif-4273. La Ferrassie, J

$26,750 \pm 250$

Bones from Level J, Late Aurignacian II industry assoc.

Gif-2427. La Ferrassie, K 3 a

$28,800 \pm 1500$

Charcoal from Level K 3 a, Aurignacian II industry assoc. Comment: undersized sample.

Gif-4274. La Ferrassie, $\mathrm{K} 2$ $27,500 \pm 280$

Bones from Level K 2, assoc Aurignacian II industry.

Gif-2428. La Ferrassie, K 2/3

$15,180 \pm 130$

Bones from Level K 2/3, assoc Aurignacian II industry.

Gif-4275. La Ferrassie, K 3 b

$27,100 \pm 320$

Bones from Level K 3 b; assoc Aurignacian II industry.

Gif-4277. La Ferrassie, K 4

$31,300 \pm 300$

Bones from Level K 4, assoc Aurignacian II industry.

Gif-4278. La Ferrassie K 5

$\geq 31,250$

Bones from Level K 5 , assoc Late Aurignacian industry. 
Gif-2423. La Ferrassie, K 6

Bones from Level K 6, assoc Aurignacian I industry. Comment: date is aberrant.

Gif-4279. La Ferrassie, $\mathrm{K} 6$

Bones from Level K 6, assoc Aurignacian I industry.

Gif-4584. La Ferrassie, M 2 e I

Long bones from Level M 2 e, assoc Mousterian industry.

Gif-4584bis. La Ferrassie, M 2 e II

Carbonate fraction of long bones, Gif-4584. Comment: date shows contamination.

Gif-4584 IV. La Ferrassie, M 2 e III

$10,800 \pm 120$

Organic fraction from long bones, Gif-4584, obtained during first $\mathrm{HCl}$ treatment of bones, in order to destroy mineral fraction before extraction of "collagen" fraction.

Gif-4584ter. La Ferrassie, M 2 e IV

$\geq 36,000$

Ethmoid bones from Level M 2 e, assoc Mousterian industry.

Gif-4584V. La Ferrassie, M 2 e V

$5820 \pm 120$

Carbonate fraction of ethmoid bones, Gif-4584ter.

Gif-4583bis. La Ferrassie, M 2 c I

$3300 \pm 100$

Carbonate fraction of bones from Level M 2 c. Insufficient collagen for dating; Mousterian industry assoc.

Gif-4583IV. La Ferrassie, M 2 c II

$17,800 \pm 400$

Organic fraction obtained after first $\mathrm{HCl}$ treatment of bones from Level M 2 c, as for Gif-4584IV.

Gif-4583ter. La Ferrassie, M 2 c III

$18,040 \pm 230$

Total organic fraction obtained after slow destruction of mineral fraction of bones by $\mathrm{HCl}$ treatment.

Gif-4585. La Ferrassie, L 3 b I

$24,300 \pm 400$

Bones from Level L 3 b, assoc Mousterian industry.

Gif-4585bis. La Ferrassie, L 3 b II

$18,000 \pm 500$

Organic fraction of bones from level L 3 b obtained after first HCL treatment, as Gif-4584IV.

General Comment: some of these dates are too young because of recent contamination of bones in situ, which shows importance of this effect on old bones. Thus, all dates might be suspect, yet older age obtained for each 
level could be best. Some dates for Aurignacian V, IV, and III are quite coherent with archaeol. Gif-2427 gives good date for charcoal from Aurignacian II level; it is ref age for better chronology of site.

Gif-2418. Gironde estuary

$1120 \pm 90$

Wood from piece of wreckage in mud, Gironde estuary $\left(45^{\circ} 30^{\prime} \mathrm{N}, 0^{\circ}\right.$ $\left.20^{\prime} \mathrm{W}\right)$. Coll and subm 1971 by M Colle, Mus Royan, Charente.

\section{Gif-3597. Roquefort, Lugasson, Gironde}

$3960 \pm 140$

Charcoal from Neolithic grave in occupation level of Roquefort site $\left(44^{\circ} 45^{\prime} \mathrm{N}, 0^{\circ} 10^{\prime} \mathrm{W}\right)$. Coll and subm 1975 by J Roussot-Laroque. Comment: younger than expected; dated level is attributed to "Civilisation des Matignons" (Middle-Late Neolithic).

Gif-2258. Rhodes II, Arignac, Ariège

$12,160 \pm 160$

Bones from Hearth 5 in Layer 2, in Rhodes II rockshelter $\left(42^{\circ} 51^{\prime} \mathrm{N}\right.$, $1^{\circ} 36^{\prime} \mathrm{W}$ ). Coll and subm 1971 by R Simmonet, Foix, Ariège. Comment: dates assoc industry which corresponds to transition between Late Magdalenian and Azilian culture (Clottes \& Simmonet, 1977; Simmonet, 1967).

Gif-2513. Lons, Pyrénées Atlantiques

$2100 \pm 70$

Charcoal from Barrow 4, Lons (43 $\left.20^{\prime} \mathrm{N}, 0^{\circ} 23^{\prime} \mathrm{W}\right)$. Coll 1969 and subm 1972 by J Seigne, Dir Antiquités Hist Aquitaine, Bordeaux. Assoc with artifacts of Hallstatt period. Comment: younger than expected.

Gif-2514. Artix, Pyrénées Atlantiques

$4170 \pm 80$

Charcoal from Barrow 1, Artix (43 24' N, $\left.0^{\circ} 34^{\prime} \mathrm{W}\right)$. Coll 1970 and subm 1972 by J Seigne. Comment: dates typical Neolithic ceramics, "Pot de Fleurs," from that region.

Gif-2515. Lescar, Pyrénées Atlantiques

$3840 \pm 80$

Charcoal from Barrow VI, Lescar (43 $\left.20^{\prime} \mathrm{N}, 0^{\circ} 24^{\prime} \mathrm{W}\right)$. Coll 1968 and subm 1972 by J Seigne. Comment: same ceramics as Artix.

Gif-2516. Sauvagnon, Pyrénées Atlantiques $\quad \mathbf{3 6 2 0} \pm \mathbf{8 0}$

Charcoal from Barrow II, Sauvagnon $\left(43^{\circ} 22^{\prime} \mathrm{N}, 0^{\circ} 23^{\prime} \mathrm{W}\right)$. Coll 1970 and subm 1972 by J Seigne. Comment: dates assoc Polypod vases.

\section{Port-Leucate series, La Corrège, Aude}

Samples from important Cardial site submerged by water, Port-Leucate $\left(42^{\circ} 50^{\prime} \mathrm{N}, 3^{\circ} 00^{\prime} \mathrm{E}\right)$. Coll and subm 1972 by R Montjardin, Sete.

Gif-2747. Port-Leucate Point I A

$5410 \pm 140$

Carbonized wood.

Gif-2748. Port-Leucate Point I B

$3210+110$

Charcoal. 
Gif-2749. Port-Leucate Point 2

Charcoal.

General Comment: good agreement with assoc Cardial Neolithic ceramics. Validity of Sample I B, coll in superficial layer, is questionable.

\section{Settiva series, Petreto-Bicchisano, Corsica}

Samples from burial under Bronze age dolmen, Settiva $\left(41^{\circ} 47^{\prime} \mathrm{N}, 8^{\circ}\right.$ $57^{\prime}$ E). Coll and subm 1972 by R Grosjean, CNRS, Sartène, Corsica.

Gif-2566. Settiva, 1

$2320 \pm 100$

Charcoal, $0.5 \mathrm{~m}$ depth in level with vases.

Gif-2567. Settiva, 2

$1510 \pm 100$

Charcoal, $0.4 \mathrm{~m}$ depth, above level with vases.

Gif-2870. Settiva, 4

Bones from burial.

General Comment: dates re-use of monument and obvious disturbance of site.

\section{Araguina-Sennola series, Bonifacio, Corsica}

Charcoal from Early Neolithic site under rockshelter at Araguina-Sennola $\left(41^{\circ} 23^{\prime} \mathrm{N}, 9^{\circ} 10^{\prime} \mathrm{E}\right)$. Coll and subm 1971 by $\mathrm{F}$ de Lanfranchi and M C Weiss, Inst Corse Etudes Préhist, Ajaccio (Gagnière et al, 1969).

Gif-2324. Araguina-Sennola, Level XVII e

$6430 \pm 140$

Hearth F 3', depth $425 \mathrm{~cm}$. Comment: date agrees with assoc Early Mediterranean Neolithic burial.

Gif-2325. Araguina-Sennola, Level XVII c

$6650 \pm 140$

Hearth overlying burial, depth $392 \mathrm{~cm}$, assoc with Cardial ceramics. Comment: same age as Gif-2324, in limits of statistical error.

Gif-2705. Araguina-Sennola, Level XVIII a

$8520 \pm 150$

Hearth, in level with lithic industry but without ceramics, at $5 \mathrm{~m}$ depth under Level XVII e.

\section{Syam-Crans series, Jura}

Syam-Crans site, S Champagnole $\left(46^{\circ} 44^{\prime} \mathrm{N}, 5^{\circ} 55^{\prime} \mathrm{E}\right)$ was suggested by $\mathrm{M}$ Berthier, Conservateur Mus Nat, Paris, as possible site of Alesia Battle of Caesar vs Vercingetorix, 52 BC. Charcoal coll and subm 1972 by $\mathrm{M}$ Berthier.

Gif-2601. Syam-Crans A

$$
840 \pm 90
$$

Gif-2622. Syam-Crans B

$$
710 \pm 90
$$

General Comment: dates do not support hypothesis. 
Syam series, Jura

Samples in pits, from “Grange d'Aufferin" site, Syam $\left(46^{\circ} 42^{\prime} \mathrm{N}, 4^{\circ} 03^{\prime}\right.$

E). Coll and subm 1972 by B Edeine.

Gif-2725. Syam 1

$850 \pm 90$

Wood.

Gif-2726. Syam 2

$1200 \pm 100$

Charcoal.

General Comment: dates are coherent with assoc ceramics.

Gif-2727. Crans, Jura

$980 \pm 90$

Charcoal in Tumulus I, Crans $\left(46^{\circ} 43^{\prime} \mathrm{N}, 5^{\circ} 58^{\prime} \mathrm{E}\right)$. Coll and subm 1972 by B Edeine; depth $0.6-0.85 \mathrm{~m}$. Comment: date does not confirm Roman occupation of site, as expected.

Gif-2301. Boissia, Jura, VI b

$1900 \pm 90$

Charcoal from Late Hallstatt age, Boissia $\left(46^{\circ} 34^{\prime} \mathrm{N}, 5^{\circ} 45^{\prime} \mathrm{E}\right)$. Coll and subm 1971 by $P$ Pétrequin, Dir Antiquités Préhist Franche-Comté, Besançon. Comment: date is younger than expected.

Gif-2639. Besançon, Saint-Paul, Jura

$2550 \pm 110$

Charcoal from potter's kiln, 6, N III, Besançon $\left(47^{\circ} 14^{\prime} \mathrm{N}, 6^{\circ} 02^{\prime} \mathrm{E}\right)$. Coll and subm 1972 by P Petrequin. Comment: good date for Middle Hallstatt of assoc Saint-Paul ceramics. Expected age: 500 to 550 BC.

Gif-2656. Dampierre, Doubs

$2740 \pm 110$

Charcoal from Late Bronze age settlement site, Dampierre-sur-leDoubs $\left(47^{\circ} 29^{\prime} \mathrm{N}, 6^{\circ} 46^{\prime} \mathrm{E}\right)$. Coll and subm 1972 by P Pétrequin. Comment: date agrees with archaeol (Pétrequin, Urlacher, \& Vuaillat, 1969).

\section{Chalain Lake series, Jura}

Samples from lacustrine stas, Chalain Lake $\left(46^{\circ} 40^{\prime} \mathrm{N}, 5^{\circ} 47^{\prime} \mathrm{E}\right)$. Coll and subm 1972 and 1977 by P Pétrequin.

Gif-2637. Chalain, No. 1

$4220 \pm 140$

Fragment of wood stake, from submerged Sta 1. Comment: dates set of ceramics and lacustrine Neolithic village.

Gif-2638. Chalain, No. 2

$4280 \pm 140$

Fragment of wood stake, from submerged Sta 2. Comment: dates palisade between Neolithic and Late Bronze III villages.

Gif-4369. Chalain, Marigny

$4400 \pm 110$

Piece of wood from littoral village, Marigny, Ilôt de la Prise d'Eau. 
Gif-2553. Grotte de Nevy-sur-Seille, Jura

Wood from fossil tree trunk, revealed after rocks fell from roof of karstic cave, Nevy-sur-Seille ( $\left.46^{\circ} 45^{\prime} \mathrm{N}, 5^{\circ} 36^{\prime} \mathrm{E}\right)$. Coll and subm 1972 by $\mathrm{P}$ Bichet, Pontarlier.

\section{Spain}

\section{La Cueva del Nacimiento series, Pontones, Jaen}

Charcoal from La Cueva del Nacimiento, Pontones $\left(38^{\circ} 05^{\prime} \mathrm{N}, 2^{\circ} 18^{\prime}\right.$ W). Coll and subm 1971 by G Rodriguez, Agde, Hérault. Except for Gif5421 and -5422 , coll and subm 1980 by Pilar Lopez, Mus Arquaeol Natl, Madrid.

Gif-2367. La Cueva del Nacimiento, Level A, Layer I, 1

$840 \pm 90$

Middle Neolithic level. Comment: date conflicts with expected age probably because this superficial level was contaminated.

Gif-5421. La Cueva del Nacimiento, Level A, Layer I, 2

Middle Neolithic level.

Gif-5422. La Cueva del Nacimiento, Level A, Layer II

Early Neolithic level.

Gif-2368. La Cueva del Nacimiento, Level A, Layer II, 2

Early Neolithic level.

Gif-3741. La Cueva del Nacimiento, Level B, Layer III

$7620 \pm 140$

Mesolithic level.

Gif-3742. La Cueva del Nacimiento, Level C, Layer IV

$11,200 \pm 200$

Late Paleolithic level.

General Comment: precisely dates lasting occupation of site.

\section{Portugal}

La Gruta Nova series, Bombarral, Estramadure

Carbonaceous earth from hearths in Mousterian levels, La Gruta Nova $\left(39^{\circ} 15^{\prime} \mathrm{N}, 9^{\circ} 09^{\prime} \mathrm{E}\right)$. Coll and subm 1972 by J Roche, CNRS, Paris.
Gif-2703. La Gruta Nova, Level 16
$\mathbf{2 6 , 4 0 0} \pm 750$
Gif-2704. La Gruta Nova, Level 20
$28,900 \pm 950$ 
General Comment: dates are evidently too young; should be considered lower limit of ages.

\section{Greece}

\section{Grotte de Kitsos series, Laurion}

Samples from Neolithic Kitsos cave $\left(37^{\circ} 44^{\prime} \mathrm{N}, 21^{\circ} 41^{\prime} \mathrm{E}\right)$. Coll and subm 1972 by N Lambert, CNRS, Paris, to complete study of site (see R, 1974 , v 16, p 54-55, for first series).

\section{Gif-2538. Grotte de Kitsos}

$5950 \pm 150$

Charcoal, Ref B II, d9, Level 3.

Gif-2539. Grotte de Kitsos

$5840 \pm 150$

Charcoal, Ref B I, b 7, Level 4.

Gif-2541. Grotte de Kitsos

$5680 \pm 150$

Charcoal, Ref B II, c 8, Level 7.

General Comment: dates agree with previous ones.

\section{Dikili Tash series, Macedonia}

Charcoal from Middle Neolithic levels of Dikili Tash site $\left(41^{\circ} 00^{\prime} \mathrm{N}, 24^{\circ}\right.$ $15^{\prime}$ E). Coll and subm 1972 by J Deshayes, Univ Paris I.

Gif-2627. Dikili Tash, DT 1972, No. 1 $6370 \pm 170$

$2.5 \mathrm{~m}$ depth.

Gif-2630. Dikili Tash, DT 1972, No. 4

Gif-2628. Dikili Tash, DT 1972, No. 2

$3 \mathrm{~m}$ depth.

Gif-2629. Dikili Tash, DT 1972, No. 3

$6250 \pm 160$

$3 \mathrm{~m}$ depth; same level as Gif-2628. Comment: too young, unexplained result.

General Comment: dates agree with those already obtained for site (R, 1974, v 16, p 53$)$.

\section{Gif-2452. Mallia, Crete}

$3060 \pm 100$

Charcoal from burning level, Mallia $\left(35^{\circ} 15^{\prime} \mathrm{N}, 25^{\circ} 30^{\prime} \mathrm{E}\right)$. Coll and subm 1972 by R Treuil, Ecole Fr Athènes. Comment: disagrees with expected age, $1800 \mathrm{BC}$; unexplained result.

\section{Egypt}

\section{Ramses II series}

Two samples of wrapping coll 1977 on mummy of Ramses II while at Mus de l'Homme, Paris, for restoration. Subm by L Balout, Inst Anthropol 
Humaine, Paris, to establish different ages of two pieces of wrappings, one of which allegedly came from restoration shortly after mummification. Dated wrappings, more or less tinged with yellow-brown, were well-preserved pieces of weaving. Coloring was extracted by repeated pretreatments with chloroform.

Gif-4018. Ramses II, 36

$3040 \pm 60$ BC.

From bundle of wrappings under heel. Comment: cal ca 1340-1370

Gif-4019. Ramses II, 17

$2840 \pm 60$ $70 \mathrm{BC}$.

From bundle of wrappings in abdomen. Comment: cal ca 1030-1100+

General Comment: difference of these samples indicates that mummy was restored shortly after initial inhumation, as expected. Gif-4018 must be taken as ${ }^{14} \mathrm{C}$ age of mummy.

\section{Tunisia}

Gif-5115. Bir Oum Ali, Gabès

$14,370 \pm 110$

Ostrich egg shell from snail midden, at Bir Oum Ali, Gabès. $\left(37^{\circ} 07^{\prime} \mathrm{N}\right.$, $9^{\circ} 10^{\prime} \mathrm{E}$ ). Coll by M Harbi and subm 1976 by G Camps, Aix-en-Provence.

\section{Bir Oum Ali series}

Shell (Helix) from Capsian site, Bir Oum Ali $\left(34^{\circ} 07^{\prime} \mathrm{N}, 9^{\circ} 10^{\prime} \mathrm{E}\right)$. Coll by M Riaji and subm 1976 by $\mathrm{G}$ Camps.

Gif-4057. Level 1

$$
\begin{array}{r}
5600 \pm 150 \\
8260 \pm 180 \\
1640 \pm 90
\end{array}
$$

Gif-4058. Level 2

Gif-2770. Carthage, TU 34 RL

Wood from coastal wreckage, Carthage $\left(36^{\circ} 54^{\prime} \mathrm{N}, 10^{\circ} 16^{\prime} \mathrm{E}\right)$. Coll by diving and subm 1972 by M Amanieu, Lab Hydrobiol, Montpellier. Comment: confirms antiquity of these remains.

\section{Algeria}

Gif-5116. Oued Guettarra Cave, Bredia

Human bones from cave of Oued Guettarra $\left(35^{\circ} 46^{\prime} \mathrm{N}, 0^{\circ} 48^{\prime} \mathrm{W}\right)$. Coll 1968 and subm 1979 by G Camps. Comment: dates early Neolithic age in Algeria.

\section{Tahabort, Ahaggar series}

Charcoal from protohistoric site, Tahabort $\left(23^{\circ} 05^{\prime} \mathrm{N}, 7^{\circ} 53^{\prime} \mathrm{E}\right)$, Ahaggar massif, $2000 \mathrm{~m}$ alt, allegedly last occupation in region by "Isabaten" people. Coll by Fr-Am mission and subm 1973 by J P Maître, LAPEMO, Aix-en-Provence. 
Gif-2900. No. 519/524

0.2 to $0.5 \mathrm{~m}$ depth.

Gif-3324. No. 105

$1110 \pm 100$

0 to $0.1 \mathrm{~m}$ depth.

Gif-2898. No. 110/111

$1050 \pm 100$

0 to $0.1 \mathrm{~m}$ depth.

Gif-3325. No. 518

$930 \pm 100$

0 to $0.1 \mathrm{~m}$ depth.

Gif-3258. No. 517

$570 \pm 90$

0.10 to $0.2 \mathrm{~m}$ depth.

General Comment (JPM): recent contamination of last two samples is possibly due to animal burrows.

Gif-3408. Hassi Menikel

$5810 \pm 150$

Ostrich egg shell from Neolithic surface site, Hassi Menikel. Coll and subm 1973 by G Aumassip, CRAPE, Alger.

Gif-3409. "Le Signal"

$6080 \pm 150$

Ostrich egg shell from Neolithic surface site, Le Signal $\left(31^{\circ} 25^{\prime} \mathrm{N}, 4^{\circ}\right.$ 44' E), Sahara. Coll and subm 1973 by G Aumassip.

Gif-3410. Ouhadia

$4490 \pm 140$

Ostrich egg shell from Neolithic surface site, Ouhadia. Coll and subm 1973 by G Aumassip.

Gif-3411. Saf Saf Wadi

$5390 \pm 140$

()strich egg shell from Neolithic surface site, Saf Saf Wadi $\left(29^{\circ} 56^{\prime} \mathrm{N}\right.$, $\left.3^{\circ} 58^{\prime} \mathrm{E}\right)$. Coll and subm 1973 by G Aumassip.

\section{Ouargla series}

Ostrich egg shell in open-air surface sites, N Ouargla $\left(32^{\circ} 02^{\prime} \mathrm{N}, 5^{\circ} 15^{\prime}\right.$

E). Coll and subm 1972 by G Aumassip.

Gif-2649. "Les Burins"

$6950 \pm 170$

Gif-2650. Site 7205

$7090 \pm 170$

Gif-2651. Site 7206

$6680 \pm 170$

Gif-3412. Site 7207

$7070 \pm 170$

Gif-3413. No. AU 731

$7560 \pm 170$

General Comment: dates agree well with expected ages; industry assoc with Epipaleolithic age in region. 
Gif-2600. Iherir

Pieces of straw in wall from ruins of bldg, Iherir, Tassili-n-Ajjer $\left(25^{\circ}\right.$ $24^{\prime} \mathrm{N}, 8^{\circ} 25^{\prime} \mathrm{E}$ ). Coll and subm 1972 by H Lhote. Comment: date is surprising and unexplained.

\section{Morocco}

Gif-2420. Bouskour

$1240 \pm 90$

Wood from ancient copper mine, Bouskour. Coll and subm 1972 by M Saadi Moussa, Service Mines, Rabat, Morocco.

\section{Gif-2560. Souk Jema el Gour}

$1310 \pm 90$

Charcoal from Berber protohistoric tomb, Souk Jema el Gour ( $33^{\circ} 51^{\prime}$ N, $5^{\circ} 18^{\prime}$ W). Coll 1960 and subm 1972 by G Camps, Univ Provence, Aixen-Provence.

\section{Gif-2652. Tarfaya}

$2790 \pm 110$

Ostrich egg shell with antelope engraving, from site on dune, near Tarfaya $\left(27^{\circ} 51^{\prime} \mathrm{N}, 12^{\circ} 31^{\prime} \mathrm{W}\right)$. Coll and subm 1972 by D Grebenart, LAPEMO, Aix-en-Provence. Comment: does not confirm Epipaleolithic age expected from part of industry.

\section{Mauritania}

\section{Drayja Malichigdane series}

Samples from Neolithic sites on dunes, near Akjoujt $\left(19^{\circ} 19^{\prime} \mathrm{N}, 14^{\circ} 30^{\prime}\right.$ W). Coll and subm 1972 by J P Carbonnel, Univ Paris VI.

Gif-2550. MAU 72-25

Organic remains in ceramic vessels.

Gif-2551. MAU 72-44

Human bones.

Gif-2552. MAU 72-46

$4850 \pm 130$

Organic remains in ceramic vessels.

\section{Gif-2769. Chigettomi, R'Kiz Massif}

$1400 \pm 90$

Human bones from Medieval site under rock shelter $\left(17^{\circ} 25^{\prime} \mathrm{N}, 10^{\circ} 26^{\prime}\right.$ W). Coll and subm 1973 by Richir, French Archeol Mission in Mauritania, Bordeaux. Comment: date indicates pre-Islamic site, as expected.

\section{Senegal}

\section{Dioron Boumak series}

Samples from Shell Midden C in Saloum delta $\left(13^{\circ} 50^{\prime} \mathrm{N}, 16^{\circ} 30^{\prime} \mathrm{W}\right)$. Accumulation of shells (mainly Arca senilis) in this part of delta forms artificial island, surface 10ha. Coll and subm 1972 by C Descamps, Fac Sci, Perpignan. 
Gif-2712. Dioron Boumak

Otholites, $1.5 \mathrm{~m}$ depth.

Gif-2711. Dioron Boumak

$810 \pm 90$

Human bones, $1.7 \mathrm{~m}$ depth.

Gif-2713. Dioron Boumak

$850 \pm 90$

Charcoal, $2.2 \mathrm{~m}$ depth.

General Comment: Gif-2712 is corrected for $\delta^{13} \mathrm{C}$ but not for apparent age of sea water; thus, it appears too old. Dates agree with very rapid accumulation rate of these shells, which was measured $10 \mathrm{~m}$ in $500 \mathrm{yr}$ in another part of island (Descamps, Thilmans, \& Thommeret, 1974).

Gif-2508. N'Dalane

$4770 \pm 120$

Charcoal from Neolithic site of N'Dalane, at 330 to $360 \mathrm{~cm}$ depth. Coll and subm 1971 by Cyr Descamps.

Niger

\section{Tezamak series, Aïr}

Charcoal from ruins of Tezamak, $50 \mathrm{~km} \mathrm{N-NE}$ of Agadez. Coll and subm 1974 by H Lhote, Mus Homme, Paris.

Gif-3525. Tezamak 1

$400 \pm 80$

In refuse pit.

Gif-3526. Tezamak 2

$330 \pm 80$

In blacksmith hearth.

Gif-3527. Aghroum Balkarène, Aïr

$\mathbf{2 0 0} \pm \mathbf{8 0}$

Charcoal in refuse pit from ruins of village, Aghroum Balkarène, $32 \mathrm{~km}$ ENE of Agadez $\left(17^{\circ} 00^{\prime} \mathrm{N}, 7^{\circ} 56^{\prime} \mathrm{E}\right)$. Coll and subm 1974 by $\mathrm{H}$ Lhote.

Gif-3522. Anisamane, Aïr

$230 \pm 80$

Charcoal in cooking pot with food remains, from ruins of Anisamane, $31 \mathrm{~km}$ NW of Agadez. Anisamane was ancient capital of Aïr. Coll and subm 1974 by H Lhote.

\section{Tadeïni series, Aïr}

Charcoal from blast furnace in Medieval village, Tadeïni, $15 \mathrm{~km} \mathrm{E}$ of Jola, Aïr. Coll and subm 1974 by H Lhote.

Gif-3523. Tadeïni 1

$$
250 \pm 80
$$

Gif-3524. Tadeïni 2

$350 \pm 80$

\section{Tadeliza series}

Charcoal from ruins of citadel, Tadeliza, Aïr $\left(17^{\circ} 05^{\prime} \mathrm{N}, 8^{\circ} 03^{\prime} \mathrm{E}\right)$. Coll and subm 1972 by H Lhote. 
Gif-2593. Tadeliza, surface

Gif-2594. Tadeliza

30 to $40 \mathrm{~cm}$ depth.

General Comment: dates agree with expected age.

\section{Marandet series}

Charcoal from forge workshops, Marandet, Aïr $\left(16^{\circ} 23^{\prime} \mathrm{N}, 7^{\circ} 25^{\prime} \mathrm{E}\right)$. Coll and subm 1972 by H Lhote.

Gif-2595. Marandet, Site A $1430 \pm 100$

Gif-2596. Marandet, Site D $1360 \pm 100$ 20 to $40 \mathrm{~cm}$ depth.

Gif-2597. Marandet, Site D $1310 \pm 100$ 100 to $150 \mathrm{~cm}$ depth.

Gif-2598. Marandet, Site $O$ $1160 \pm 100$ 5 to $20 \mathrm{~cm}$ depth.

Gif-2599. Marandet, Site G $1420 \pm 100$ $25 \mathrm{~cm}$ depth.

General Comment: this important forge where 30,000 crucibles were found, is dated to 6 th century AD.

Gif-3516. Tiguermaouine, Aïr

$4220 \pm 110$

Charcoal near skeleton of woman dressed in leather with child, in Neolithic site, Tiguermaouine, $30 \mathrm{~km} \mathrm{~N}$ of Arlit $\left(17^{\circ} 00^{\prime} \mathrm{N}, 7^{\circ} 56^{\prime} \mathrm{E}\right)$. Coll and subm 1974 by $\mathrm{H}$ Lhote.

Gif-3517. Taoulaoualt, Aïr

$4150 \pm 110$

Charcoal with ceramics in Neolithic kiln, Taoulaoualt, similar to those found at Arlit. Coll and subm 1974 by $\mathrm{H}$ Lhote.

\section{Arlit series, Aïr}

Samples from Neolithic sites of Arlit $\left(18^{\circ} 44^{\prime} \mathrm{N}, 7^{\circ} 43^{\prime} \mathrm{E}\right)$. Coll and subm 1973-1974 by H Lhote.

Gif-2933. Arlit 2

$4650 \pm 130$

Charcoal, scattered in ground, $1.5 \mathrm{~m}$ depth.

Gif-2934. Arlit 3

$5240 \pm 140$

Charcoal, $1.5 \mathrm{~m}$ depth.

Gif-2935. Arlit 4

$2290 \pm 110$

Piece of carbonized wood, Sq I, $1.65 \mathrm{~m}$ depth. 
Gif-2937. Arlit 5

$4040 \pm 110$

Mussel shell fragments, Sq III, between surface and $1 \mathrm{~m}$ depth.

Gif-3057. Arlit

$5380 \pm 130$

Organic matter from habitation soil, 1.92 to $1.97 \mathrm{~m}$ depth, in contact with skeleton.

Gif-3518. Arlit I

$4840 \pm 130$

Charcoal, 1.8 to $2.2 \mathrm{~m}$ depth.

Gif-3519. Arlit II

$4960 \pm 130$

Charcoal between surface and $1 \mathrm{~m}$ depth.

Gif-3521. Arlit IV

$4950 \pm 150$

Bone fragments, Sq 25-27, between surface and $1 \mathrm{~m}$ depth.

General Comment: mussel shells indicate existence of permanent rivers in present desert and abundant lithic and ceramic industries indicate important human occupation; also, favorable climatic conditions between 4000 and $5000 \mathrm{BP}$ in Arlit region.

Gif-2938. Ifanghalene, Aïr

$3410 \pm 100$

Mussel shells from Ifanghalene, $5 \mathrm{~km}$ W of Arlit. Coll and subm 1973 by $\mathrm{H}$ Lhote.

Gif-2936. Tibakaratin, Aïr

$4540 \pm 130$

Charcoal from Neolithic site of Tibakaratin, SW of Arlit. Coll and subm 1973 by H Lhote.

Gif-2939. In-Gall

$\geq 38,000$

Carbonized wood, found during well digging, In-Gall region $\left(16^{\circ} 51^{\prime}\right.$ N, $\left.7^{\circ} 01^{\prime} \mathrm{E}\right), 40 \mathrm{~m}$ depth. Subm 1973 by H Lhote.

\section{Chad}

\section{Koro Toro series}

Charcoal from Iron age mounds in Koro Toro region. Coll and subm 1972-73-77 by F Treinen-Claustre, CNRS, Toulouse.

Gif-2613. Nemra

$730 \pm 90$

0.1 to $0.3 \mathrm{~m}$ depth $\left(16^{\circ} 17^{\prime} \mathrm{N}, 18^{\circ} 33^{\prime} \mathrm{E}\right)$, from Late Iron age.

Gif-2611. Bochianga 5A

$1500 \pm 100$

$0.8 \mathrm{~m}$ depth at Bochianga $\left(16^{\circ} 06^{\prime} \mathrm{N}, 18^{\circ} 26^{\prime} \mathrm{E}\right)$.

Gif-2612. Bochianga 5B

$1500 \pm 100$

$1.35 \mathrm{~m}$ depth.

Gif-2895. Bahali

$630 \pm 90$

0.15 to $0.2 \mathrm{~m}$ depth at Bahali $\left(16^{\circ} 13^{\prime} \mathrm{N}, 18^{\circ} 26^{\prime} \mathrm{E}\right)$ 
Gif-4201. Bahali, Site 110

$1540 \pm 90$

$0.2 \mathrm{~m}$ depth.

Gif-2896. Toungour Salado

$1340 \pm 100$

$0.3 \mathrm{~m}$ depth.

Gif-4202. Kebir Rosa A, Site 34

$2360 \pm 100$

0.1 to $0.15 \mathrm{~m}$ depth, Kebir Rosa $\left(16^{\circ} 05^{\prime} \mathrm{N}, 18^{\circ} 45^{\prime} \mathrm{E}\right)$.

Gif-4193. Kebir Rosa B, Site 34

$1480 \pm 100$

0.15 to $0.2 \mathrm{~m}$ depth.

Gif-4199. Koro Toro A, Site 4

$1170 \pm 90$ mound.

0.1 to $0.15 \mathrm{~m}$, Koro Toro $\left(16^{\circ} 05^{\prime} \mathrm{N}, 18^{\circ} 45^{\prime} \mathrm{E}\right)$ from iron workshop on

Gif-4198. Koro Toro B, Site 4

$1230 \pm 100$

$0.35 \mathrm{~m}$ depth.

Gif-4194. Koro Toro C, Site 4

$1580 \pm 100$

$0.8 \mathrm{~m}$ depth.

Gif-4195. Koro Toro E, Site 4

$1400 \pm 100$

$2.2 \mathrm{~m}$ depth.

Gif-4196. Krimé A, Site 3

$670 \pm 100$

$0.3 \mathrm{~m}$ depth, Krimé $\left(15^{\circ} 05^{\prime} \mathrm{N}, 18^{\circ} 28^{\prime} \mathrm{E}\right)$ from iron workshop on mound.

Gif-4197. Krimé B, Site 3

$1410 \pm 100$

$1 \mathrm{~m}$ depth.

Gif-4200. Goz Kerki, Site 97

$1250 \pm 90$

$0.2 \mathrm{~m}$ depth.

General Comment: dates Iron age in Chad; oldest site dated to 5 th century BC.

\section{Messo series}

Charcoal from habitation mound, Messo, site of type Sao III, $58 \mathrm{~km}$ NNW of Fort-Lamy $\left(12^{\circ} 10^{\prime} \mathrm{N}, 14^{\circ} 59^{\prime} \mathrm{E}\right)$. Coll and subm 1973 by $\mathrm{J} \mathrm{P}$ Lebeuf, CNRS, Paris.

Gif-2784. Messo, Pt III, Level 2

$260 \pm 90$

$0.6 \mathrm{~m}$ depth.

Gif-2786. Messo, Pt III, Level 8

$320 \pm 90$

2.5m depth. 
Gif-2785. Messo, Pt III, Level 9

$320 \pm 90$

$2.7 \mathrm{~m}$ depth.

Gif-2787. Messo, Pt IV

Modern

$0.5 \mathrm{~m}$ depth beside skeleton. Comment: recent burial in archaeol site.

\section{Cameroon}

\section{Sou series}

Charcoal from habitation mound, Sou, site of type Sao II $\left(12^{\circ} 12^{\prime} \mathrm{N}\right.$, $14^{\circ} 42^{\prime} \mathrm{E}$ ), Logone-et-Chari. Coll and subm 1977-1979 by J P Lebeuf, M D Lebeuf, and J Rapp.

Gif-4150. Sou, Pt I, 103 $150 \pm 80$

$1.7 \mathrm{~m}$ depth.

Gif-4149. Sou, Pt I, 118

$580 \pm 80$

$1.9 \mathrm{~m}$ depth.

Gif-4152. Sou, Pt II, 392

$520 \pm 80$

$0.5 \mathrm{~m}$ depth.

Gif-4148. Sou, Pt III, 102

Modern

$0.6 \mathrm{~m}$ depth.

Gif-4151. Sou, Pt XI, 330

$650 \pm 80$

Level II.

Gif-4504. Sou, Pt XI, 1221

$620 \pm 80$

Under Gif-4151.

Gif-4932. Sou, Pt XI, 1756

$850 \pm 90$

$4.2 \mathrm{~m}$ depth.

Gif-4933. Sou, Pt XX, 1732

$1340 \pm 90$

$7.3 \mathrm{~m}$ depth.

\section{Sou Blame Radjil series}

Charcoal from habitation mound, Sou Blame Radjil, site of type Sao I $\left(12^{\circ} 13^{\prime} \mathrm{N}, 14^{\circ} 42^{\prime} \mathrm{E}\right)$. Coll by J Rapp and subm 1978 by J P Lebeuf.

Gif-4820. Sou Blame, 168-6

$500 \pm 60$

Level $1 \mathrm{a}, 0.49 \mathrm{~m}$ depth.

Gif-4821. Sou Blame, 114

$2340 \pm 1100$

Level $3 \mathrm{a}, 2.78 \mathrm{~m}$ depth.

Gif-4934. Sou Blame, 168-329

$2800 \pm 110$

Level 6, $4 \mathrm{~m}$ depth. 
General Comment: dates agree well with other results for Sou Blame: for Level 3a, $2310 \pm 150$ : Ly-2003, $2280 \pm 170$ : Ly-2004, and for Level 4/5, $2530 \pm 120:$ Ly-2005 (R, 1983, v 25, p 91). Dates from Sou Blame, Sou and Masso mounds, as well as previous results from similar mound, Mdaga (R, 1972, v 14, p 294) establish chronology of Sao culture from 9 th century BC to 19 th century AD.

\section{Argentina}

\section{Quimili Paso series}

Charcoal from Quimili Paso site, Prov Santiago del Estero $\left(28^{\circ} 30^{\prime} \mathrm{S}\right.$, $63^{\circ}$ W). Coll 1968 and subm 1971 by A M Lorandi, Mus Ciencas Nat, La Plata. Ceramics belong to Middle Sunchituyoj culture.

Gif-2308. Quimili Paso, QP SICI, 2 $670 \pm 60$ At depth $0.3 \mathrm{~cm}$.

Gif-2309. Quimili Paso, QP SICI, 3 $730 \pm 60$ At depth $0.45 \mathrm{~cm}$.

Gif-2310. Quimili Paso, QP SICI, 4 $590 \pm 60$ At depth $0.6 \mathrm{~cm}$.

General Comment: dates are consistent with archaeol data.

Brazil

Gif-3223. Toca do Gongo, Piaui

Charcoal from Burial 3 in rock shelter, Toca do Gongo $\left(9^{\circ} \mathrm{S}, 42^{\circ} \mathrm{W}\right)$. Coll by N Guidon and subm 1974 by A Emperaire, CNRS, Paris.

Gif-3225. AQN, Piaui

$1690 \pm 110$

Charcoal from AQN village $\left(9^{\circ} \mathrm{S}, 42^{\circ} \mathrm{W}\right)$, level 20 to $30 \mathrm{~cm}$ depth. Coll by $\mathrm{N}$ Guidon and subm 1974 by A Emperaire.

Gif-3909. Toca do Bananeira, Goias

Charcoal from rock shelter Toca do Bananeira $\left(15^{\circ} 57^{\prime} \mathrm{S}, 50^{\circ} 07^{\prime} \mathrm{W}\right)$, $200 \mathrm{~km}$ from Brasilia. Coll and subm 1976 by A Emperaire. Assoc with fine industry of chipped stones.

Gif-3910. Gruta do Salitre, Goias

$1230 \pm 90$

Charcoal in cave, Gruta do Salitre $\left(15^{\circ} 57^{\prime} \mathrm{S}, 50^{\circ} 07^{\prime} \mathrm{W}\right), 200 \mathrm{~km}$ from Brasilia. Coll and subm 1976 by A Emperaire. Assoc with fine industry of chipped stones.

\section{Lapa Vermelha IV series, Minas Gerais, Lagoa Santa}

Charcoal samples from rockshelter site of Lapa Vermelha IV $\left(19^{\circ} 40^{\prime}\right.$ S, 43 $54^{\prime}$ W) (Laming-Emperaire, 1979). Coll and subm 1972-73-75-76 by A Laming-Emperaire. Shelter's walls are covered with many rupestral paintings and engravings. 
Gif-2735. Lapa Vermelha IV, base, Level I, 2001

$320 \pm 80$

At depth $0.2 \mathrm{~m}$; corresponds to appearance of ceramics, in Lagoa Santa region.

Gif-2732. Lapa Vermelha IV, B, 1008 $300 \pm 110$

At depth $1.15 \mathrm{~m}$.

Gif-2733. Lapa Vermelha IV, B, 1019

$3740 \pm 110$

At depth $1.5 \mathrm{~m}$.

Gif-2545. Lapa Vermelha IV, A, 2010

$3720 \pm 120$

Gif-2734. Lapa Vermelha IV, B, 1025

$3660 \pm 110$

At depth $2.1 \mathrm{~m}$.

Gif-2543. Lapa Vermelha IV, C, 1099

$4170 \pm 120$

At depth 4.35m, Level 7.

Gif-2544. Lapa Vermelha IV, C, 3019

$4400 \pm 120$

At depth $5 \mathrm{~m}$.

Gif-3222. Lapa Vermelha IV, Unit 23 F

$1620 \pm 100$

Base level B.

Gif-3220. Lapa Vermelha IV, Unit 25 B

$1880 \pm 140$

Surface.

Gif-3211. Lapa Vermelha IV, Unit 24 D

$3260 \pm 110$

Gif-3219. Lapa Vermelha IV, Unit 24 E

$3430 \pm 130$

Base level C.

Gif-3221. Lapa Vermelha IV, Unit 25-26-27 DEF

$3070 \pm 110$

Level D.

Gif-3218. Lapa Vermelha IV, Unit 24 D

$3370 \pm 110$

Base level D.

Gif-3210. Lapa Vermelha IV, Unit 25-26 EF

$3580 \pm 130$

Level E, Hearth 8.

Gif-3209. Lapa Vermelha IV, Unit 25 E

$3750 \pm 110$

Level E, Hearth 9.

Gif-3213. Lapa Vermelha IV, Unit 24-25 DC

$4550 \pm 130$

Level F.

Gif-3215. Lapa Vermelha IV, Unit 25 CD

$4350 \pm 120$

Level G, 1st layer. 
Gif-3214. Lapa Vermelha IV, Unit 25 CD

Level G, 3rd layer, in hearth.

Gif-3216. Lapa Vermelha IV, Unit 24-25 DCB

$8490 \pm 160$

Level $\mathrm{H}$.

Gif-3217. Lapa Vermelha IV, Unit 25 BC

$6950 \pm 140$

Level I.

Gif-3207. Lapa Vermelha IV, Unit 33 D

$6830 \pm 150$

At depth $9.65 \mathrm{~m}$.

Gif-3907. Lapa Vermelha IV, Unit 32 B, 1975-87 $\quad 5400 \pm 500$

At depth 12.95 to $13.15 \mathrm{~m}$; undersized sample.

GIF-3208. Lapa Vermelha IV, Unit 33 C

$9580 \pm 200$

At depth 10.3 to $10.8 \mathrm{~m}$.

Gif-3727. Lapa Vermelha IV, Unit 32-33, 1975-28 $10,200 \pm 220$

At depth 11.7 to $11.9 \mathrm{~m}$.

Gif-3726. Lapa Vermelha IV, Unit 27-28-29 BA, 1975-48 $\quad 11,680 \pm 500$

At depth $11.7 \mathrm{~m}$; undersized sample.

Gif-3906. Lapa Vermelha IV, Unit 32 B, 1975-48 $\quad 12,960 \pm 300$

At depth 12.6 to $12.8 \mathrm{~m}$, in red sediment.

Gif-3905. Lapa Vermelha IV, Unit 32-33 BA, 1975-121

$15,300 \pm 400$

At depth 13.55 to $14.5 \mathrm{~m}$.

Gif-3725. Lapa Vermelha IV, Unit 32-33 CB, 1975-14 $\geq 25,000$

At depth 11.7 to $11.8 \mathrm{~m}$, in yellow sediment. Comment: undersized sample; result may be less reliable than others.

Gif-3908. Lapa Vermelha IV, Unit 32-33 B

$22,410 \pm 400$

At depth 12.6 to $13.55 \mathrm{~m}$, in yellow sediment. Comment (AP): very interesting date but obtained with some mixed samples not very well identified. However, confirms antiquity of site.

General Comment: evidence of human occupation in all levels of site; presence of human skull at depth $12.9 \mathrm{~m}$.

\section{Almeida series, Piraju}

Charcoal samples from hearths in open-air site, Almeida $\left(23^{\circ} 10^{\prime} \mathrm{S}, 49^{\circ}\right.$ 21' W). Coll by L Pallestrini and subm 1973 by A Emperaire. 
Gif-2730. Almeida, Hearth A

Gif-2731. Almeida, Hearth B

Gif-2738. Fazenda, Minas Gerais 1971, Mangueira 4045 Lagoa Santa

$4670 \pm 130$

Charcoal from human occupation level, depth 0.9 to $1 \mathrm{~m}$, Minas Gerais $\left(20^{\circ} \mathrm{S}, 44^{\circ} \mathrm{W}\right)$. Coll 1971 by A Prous-Poirier and subm 1973 by A Emperaire.

Gif-2737. Caieras, Minas Gerais 1971, 4010 Lagoa Santa $9500 \pm 200$

Charcoal from rockshelter site of Caieras with rupestral paintings $\left(19^{\circ}\right.$ $32^{\prime} \mathrm{S}, 44^{\circ} 04^{\prime} \mathrm{W}$ ). Coll 1971 by A Prous-Poirier and subm 1973 by A Emperaire.

Gif-2546. Piraju, Sao Paulo

$3600 \pm 120$

Charcoal from preceramic level just underlying ceramic level, 80 to

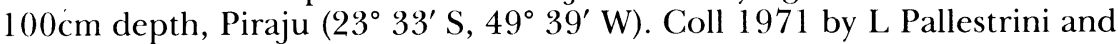
subm 1972 by A Emperaire.

\section{Itapiranga series, Santa Catarina}

Charcoal from Sec 1, Itapiranga $\left(27^{\circ} 11^{\prime} \mathrm{S}, 53^{\circ} 45^{\prime} \mathrm{W}\right)$. Coll 1968 by J A Rohr and subm 1972 by A Emperaire.

Gif-2548. Itapiranga 1-C

$7600 \pm 160$

At depth $5.3 \mathrm{~m}$.

Gif-2547. Itapiranga 1-B

$7560 \pm 160$

At depth $6 \mathrm{~m}$.

Gif-2542. Itapiranga 1-A

$8640 \pm 180$

At depth 7.3m.

\section{Peru}

Tellarmachay series, Central Andes

Charcoal from rock shelter, Tallarmachay $\left(11^{\circ} 10^{\prime} \mathrm{S}, 75^{\circ} 50^{\prime} \mathrm{W}\right)$. Coll and subm 1974-1975 by D Lavallée and M Julien, Mus Homme, Paris.

Gif-3481. Tellarmachay

$3370 \pm 180$

At depth 50 to $70 \mathrm{~cm}$; preceramic level. Comment: undersized sample.

Gif-3482. Tellarmachay

$4400 \pm 200$

At depth 70 to $130 \mathrm{~cm}$; preceramic level. Comment: undersized sample.

Gif-3772. Tellarmachay, Level II b

$2190 \pm 100$

Level with "Formative" ceramics. 
Gif-3773. Tellarmachay, Level II c

Level with "Formative" ceramics.

Gif-3774. Tellarmachay, Base level II c

$2120 \pm 100$

Level with "Formative" ceramics.

General Comment: dates "Formative ceramics" period.

Gif-3483. Ccollpa, Central Andes

$2100 \pm 150$

Charcoal from rock shelter, Ccollpa $\left(11^{\circ} 12^{\prime} \mathrm{S}, 75^{\circ} 50^{\prime} \mathrm{W}\right)$, alt + $4100 \mathrm{~m}$, depth 25 to $30 \mathrm{~cm}$. Coll and subm 1975 by M Julien. Early level with ceramic occupations. Comment: corresponds to age for "Formative" at Tellarmachay.

\section{Tantamayo series, Huamalies, Huanuco}

Charcoal assoc with lithic industry from preceramic site with stone houses, Tantamayo region $\left(9^{\circ} 05^{\prime} \mathrm{S}, 79^{\circ} \mathrm{W}\right)$. Coll 1970 and subm 1971 by $\mathrm{L}$ Girault, Mus Homme, Paris.

Gif-2306. Tantamayo 15

$3910 \pm 130$

Charcoal from Layer 2.

Gif-2302. Tantamayo 2

$3930 \pm 130$

Charcoal from Layer 3.

Gif-2305. Tantamayo 12

$3880 \pm 130$

Charcoal from Layer 3.

Gif-2303. Tantamayo 5

$4030 \pm 130$

Charcoal from Layer 4.

Gif-2304. Tantamayo 7

$4050 \pm 130$

Charcoal from Layer 4.

General Comment: dates stage of preceramic culture in Andean region of Peru.

\section{GEOLOGIC SAMPLES}

Peat Bogs

France

\section{Somme Valley series, Somme}

Freshwater peat from cores in sediment of valley. Coll and subm 1975 by M F Huault, Univ Rouen. Alts related to present msl.

Gif-3705. Roudray, R 29

$1960 \pm 100$

$+2.10 \mathrm{~m}\left(50^{\circ} 06^{\prime} \mathrm{N}, 1^{\circ} 32^{\prime} \mathrm{E}\right)$. Pollen zone: Sub-atlantic. 
$+1.30 \mathrm{~m}\left(50^{\circ} 06^{\prime} \mathrm{N}, 1^{\circ} 32^{\prime} \mathrm{E}\right)$. Pollen zone: Sub-atlantic. Comment: strongly disagrees with expected age.

Gif-3707. Erondelle, E 35

$+5.50 \mathrm{~m}\left(50^{\circ} 03^{\prime} \mathrm{N}, 1^{\circ} 27^{\prime} \mathrm{E}\right)$. Pollen zone: beginning of Sub-atlantic. Comment: much older than expected.

Gif-3708. Erondelle, E 40

$3490 \pm 100$

$+5 \mathrm{~m}\left(50^{\circ} 03^{\prime} \mathrm{N}, 1^{\circ} 27^{\prime} \mathrm{E}\right)$. Pollen zone: end of Sub-boreal.

Gif-3945. Avranches, Manche, 2740

$2320 \pm 90$

Peaty clay from core $2470,\left(48^{\circ} 42^{\prime} \mathrm{N}, 1^{\circ} 21^{\prime} \mathrm{W}\right),-3.80 \mathrm{~m}$. Pollen zone: Atlantic-Sub-boreal limit. Comment: disagrees strongly with expected age.

Gif-3946. Avranches, Manche, 2451

$3410 \pm 100$

Peaty clay from core $2451,-2.20 \mathrm{~m}$. Pollen zone: Sub-boreal.

Gif-3947. Pontorson, Manche, 2480

$3100 \pm 100$

Freshwater peat, from core $2480\left(48^{\circ} 34^{\prime} \mathrm{N}, 1^{\circ} 30^{\prime} \mathrm{W}\right),+4.80 \mathrm{~m}$. Pollen zone: Sub-atlantic.

Gif-3948. Pontorson, Manche, 2491

$5140 \pm 120$ eal.

Plant remains in clay, from core $2491,+3.80 \mathrm{~m}$. Pollen zone: Sub-bor-

Gif-2955. Wathiéhurt, Cayeux, Somme

$7540 \pm 140$

Peat from lower part of Quaternary deposits $\left(50^{\circ} 11^{\prime} \mathrm{N}, 1^{\circ} 30^{\prime} \mathrm{E}\right)$, $-10 \mathrm{~m}$. Coll and subm 1973 by P Broquet, Fac Sci, Amiens.

\section{Seine Valley series, Seine Maritime}

Samples from cores in valley and paleovalley of Seine R; subm 1973 by M F Huault. Alts are related to present msl.

Gif-2761. Core $22 \mathrm{~A}, 768$

$8200 \pm 160$

Clayey peat, $-16.72 \mathrm{~m}$. Pollen zone: end Boreal/beginning of Atlantic. Comment: date disagrees with palynology.

Gif-2762. Core 35 B, 820

$7270 \pm 140$

Saltwater peat, $-14.60 \mathrm{~m}$. Pollen zone: Atlantic.

Gif-2763. Core 4 A, 3

$\mathbf{7 7 8 0} \pm \mathbf{1 5 0}$

Clayey peat, $-12.30 \mathrm{~m}$. Pollen zone: Boreal/Atlantic limit; Pinus $34 \%$.

Gif-2764. Core 37 A, 6

$9400 \pm 170$

Clayey peat, $-13.20 \mathrm{~m}$. Pollen zone: Boreal; Pinus $97 \%$. 
Gif-2875. Core 5 A, 2

Saltwater peat, $-16.50 \mathrm{~m}$. Pollen zone: Boreal/Atlantic limit.

General Comment: dates agree with palynology, except for Gif-2761, which is too old. Gif-2762 and -2875, saltwater peat, provide good indications on sea level at 7270 and $7680 \mathrm{BP}$.

\section{Bures-sur-Dives series, Calvados}

Marsh in Dives estuary $\left(49^{\circ} 12^{\prime} \mathrm{N}, 0^{\circ} 10^{\prime} \mathrm{W}\right)$. Coll and subm 1974 by M F Huault. Alts related to present msl.

Gif-3351. Bures-sur-Dives, 1402

$3100 \pm 100$

Saltwater peat, $+0.50 \mathrm{~m}$. Pollen zone: beginning of Sub-atlantic.

Gif-3352. Bures-sur-Dives, 1404

$3020 \pm 100$

Freshwater peat, $-1.20 \mathrm{~m}$. Pollen zone: end of Sub-boreal.

Gif-3353. Bures-sur-Dives, 1406

$6220 \pm 140$

Saltwater peat, $-3.20 \mathrm{~m}$. Pollen zone: end of Atlantic.

General Comment: presence of freshwater peat at $-1.2 \mathrm{~m}$ indicates short regression during Flandrian transgression, dated 3020 BP.

\section{Orne estuary series, Calvados}

Freshwater peat from core in sediment of Orne Valley, Hérouville $\left(49^{\circ}\right.$ $06^{\prime} \mathrm{N}, 2^{\circ} 39^{\prime} \mathrm{W}$ ). Coll and subm 1975 by M F Huault. Alts related to present msl.

Gif 3747. Hérouville, 2355

$3260 \pm 100$

$-1.90 \mathrm{~m}$. Pollen zone: Sub-boreal.

Gif-3495. Hérouville, 2373

$5250 \pm 120$

$-3.50 \mathrm{~m}$. Pollen zone: Atlantic Sub-boreal limit.

Gif-3496. Hérouville, 2380

$5350 \pm 120$ zone.

$-4.40 \mathrm{~m}$. Pollen zone: Atlantic. Comment: date is too young for Atlantic

\section{Gif-3916. Genets II, Mont Saint-Michel Bay}

$8890 \pm 160$

Peaty sand from soil, under dune, near Genêts $\left(48^{\circ} 41^{\prime} \mathrm{N}, 1^{\circ} 28^{\prime} \mathrm{W}\right)$. Coll and subm 1976 by JP Lautridou, Centre Géomorphol, CNRS, Caen. Comment: does not confirm expected Alleröd age.

\section{Gif-3917. Lingreville, Manche}

$3660 \pm 110$

Charcoal from hearth, at depth $1.20 \mathrm{~m}$, in sequence of sand and peat $\left(48^{\circ} 57^{\prime} \mathrm{N}, 1^{\circ} 31^{\prime} \mathrm{W}\right)$. Coll and subm 1976 by J P Lautridou.

\section{Eastern Pyrénées}

Results establish chronology of late glacial and postglacial vegetational and climatic stages (Jalut, 1974). 


\section{Fournas Brook series, Ariège}

Bog along brook, Laurent Mts $\left(42^{\circ} 42^{\prime} \mathrm{N}, 2^{\circ} 03^{\prime} \mathrm{E}\right)$. Alt $+1510 \mathrm{~m}$. Coll and subm 1972 by G Jalut, Lab Bot, Fac Sci Toulouse.

Gif-2441. Palyn 36

Modern

Peat from 20 to $30 \mathrm{~cm}$ depth.

Gif-2440. Palyn 37

$5590 \pm 120$

Peat from 160 to $170 \mathrm{~cm}$ depth. Pollen zone: Atlantic.

Gif-2439. Palyn 38

$6200 \pm 130$

Organic debris in silty sediment, from level 200 to $210 \mathrm{~cm}$ depth. Corresponds to important phase of deforestation. Pollen zone: Atlantic.

Gif-2438. Palyn 39

$5510 \pm 120$

Silty sandy sediment, from 260 to $270 \mathrm{~cm}$ depth. Pollen zone: Atlantic. Comment: date indicates recent contamination of sample.

General Comment: human influence on vegetation becomes perceptible from end of Atlantic in upper Têt Valley.

\section{Laurenti Brook series, Ariège}

Bog along brook $\left(42^{\circ} 04^{\prime} \mathrm{N}, 2^{\circ} 01^{\prime} \mathrm{E}\right)$. Alt $+1860 \mathrm{~m}$, E Pyrénées. Coll and subm 1972 by $\mathrm{G}$ Jalut.

Gif-2466. Palyn 40

$4800 \pm 130$

Peat from 90 to $100 \mathrm{~cm}$ depth. Pollen zone: beginning of Sub-boreal.

Gif-2467. Palyn 41

$6080 \pm 140$

Peat from 190 to $200 \mathrm{~cm}$ depth. Pollen zone: Atlantic.

Gif-2468. Palyn 42

$8230 \pm 180$

Peat from 270 to $280 \mathrm{~cm}$ depth. Pollen zone: beginning of Boreal.

Gif-2469. Palyn 43

$9250 \pm 190$

Peat from 320 to $330 \mathrm{~cm}$ depth. Pollen zone: Pre-boreal.

Gif-2470. Palyn 44

$8990 \pm 190$

Peaty clay from 360 to $370 \mathrm{~cm}$ depth. Pollen zone: Alleröd. Comment: date disagrees with palynology.

\section{La Moulinasse II series, Aude}

Bog at $\mathrm{N}$ side of $\mathrm{Jau}$ Col $\left(42^{\circ} 42^{\prime} \mathrm{N}, 2^{\circ} 14^{\prime} \mathrm{E}\right)$. Alt $+1380 \mathrm{~m}$. Coll and subm 1972 by $G$ Jalut.

Gif-2657. Palyn 45

$1750 \pm 100$

Peat from 120 to $130 \mathrm{~cm}$ depth. Pollen zone: Sub-atlantic. First appearance of cultivation. 
Gif-2658. Palyn 46

Peat from 200 to $210 \mathrm{~cm}$ depth. Pollen zone: Sub-boreal.

Gif-2659. Palyn 47

$7420 \pm 160$

Peat from 220 to $230 \mathrm{~cm}$ depth. Pollen zone: beginning of Boreal. Comment: date disagrees with palynology; probably contaminated during sampling.

Gif-2660. Palyn 48

$7420 \pm 160$

Peat from 250 to $260 \mathrm{~cm}$ depth. Pollen zone: beginning of Pre-boreal. Comment: same as Gif-2659.

Gif-2661. Palyn 49

$7500 \pm 160$

Peat from 300 to $310 \mathrm{~cm}$ depth. Pollen zone: Pre-boreal. Comment: same as Gif-2659.

Gif-2662. Palyn 50

$12,140 \pm 240$ Dryas.

Organic sand from 330 to $340 \mathrm{~cm}$ depth. Pollen zone: end of Older

\section{Nohèdes series, E Pyrénées}

Above glacial moraine $\left(42^{\circ} 38^{\prime} \mathrm{N}, 2^{\circ} 14^{\prime} \mathrm{E}\right)$, alt $+1680 \mathrm{~m}$, in Bassin de La Têt R. Coll and subm 1973 by G Jalut.

\section{Gif-2982. Palyn 52}

$920 \pm 100$

Peat from 30 to $34 \mathrm{~cm}$ depth. Pollen zone: Sub-atlantic.

Gif-2983. Palyn 53

$3020 \pm 100$

Organic clay from 50 to $52 \mathrm{~cm}$ depth. Pollen zone: Sub-boreal.

Gif-2984. Palyn 54

$4540 \pm 130$ real.

Clayey sandy sediment from 74 to $76 \mathrm{~cm}$ depth. Pollen zone: Sub-bo-

Gif-2985. Palyn 55

$7920 \pm 500$

Clayey sandy sediment from 82 to $84 \mathrm{~cm}$ depth. Pollen zone: Atlantic. Comment: undersized sample.

Gif-2986. Palyn 56

$9800 \pm 100$

Sandy sediment from 107 to $109 \mathrm{~cm}$ depth. Pollen zone: Pre-boreal.

\section{Capvern series, Hautes Pyrénées}

Bog $\left(43^{\circ} 06^{\prime} \mathrm{N}, 0^{\circ} 01^{\prime} \mathrm{E}\right)$, alt +590 . Coll and subm 1976 by G Jalut.

Gif-3801. Palyn 57

$390 \pm 80$

Silty peat from 35 to $57 \mathrm{~cm}$ depth. Pollen zone: Sub-atlantic. 
Gif-3802. Palyn 58

Silty peat from 119 to $121 \mathrm{~cm}$ depth. Pollen zone: Sub-atlantic.

\section{Ossun series, Hautes Pyrénées}

Bog $\left(43^{\circ} 10^{\prime} \mathrm{N}, 0^{\circ} 03^{\prime} \mathrm{W}\right)$, alt $+375 \mathrm{~m}$. Coll and subm 1976 by $\mathrm{S}$ Omer and $\mathrm{G}$ Jalut.

\section{Gif-3803. Palyn 59}

$630 \pm 80$

Peaty clay from 51 to $59 \mathrm{~cm}$ depth. Pollen zone: Sub-atlantic.

Gif-3804. Palyn 60

$470 \pm 90$

Peaty clay from 102 to $106 \mathrm{~cm}$ depth. Pollen zone: Sub-atlantic.

\section{Lhers Pond series, Ariège}

Pond $\left(42^{\circ} 48^{\prime} \mathrm{N}, 1^{\circ} 20^{\prime} \mathrm{E}\right)$, alt $+1274 \mathrm{~m}$. Coll and subm 1976 by J V Kenla and $\mathrm{G}$ Jalut.

Gif-3911. Palyn 61

Peat from 58 to $60 \mathrm{~cm}$ depth. Pollen zone: Atlantic.

Gif-3912. Palyn 62

Peat from 148 to $150 \mathrm{~cm}$ depth. Pollen zone: Sub-atlantic.

Gif-3913. Palyn 63

Peat from 200 to $202 \mathrm{~cm}$ depth. Pollen zone: Sub-atlantic.

Gif-3914. Palyn 64

Peat from 249 to $251 \mathrm{~cm}$ depth. Pollen zone: Sub-atlantic.

Gif-3915. Palyn 65

$5360 \pm 140$

Peat from 260 to $261 \mathrm{~cm}$ depth. Pollen zone: Sub-boreal.

General Comment: observed discontinuity of sedimentation between 250 and $260 \mathrm{~cm}$ explains difference between ages for these levels.

Pla de Soulcem series, Ariège Jalut.

Bog $\left(42^{\circ} 40^{\prime} \mathrm{N}, 1^{\circ} 26^{\prime} \mathrm{W}\right)$, alt $+1525 \mathrm{~m}$. Coll and subm 1977 by $\mathrm{G}$

Gif-4385. Palyn 66

$1160 \pm 90$

Peaty sediment from 130 to $140 \mathrm{~cm}$ depth.

Gif-4587. Palyn 67

$2180 \pm 90$

Peaty sediment from 172 to $182 \mathrm{~cm}$ depth.

Gif-4386. Palyn 68

$3320 \pm 100$

Peaty sediment from 172 to $182 \mathrm{~cm}$ depth.

General Comment: abundance of herbs and Abies indicate that deforestation was almost total by $3320 \mathrm{BP}$ ( Jalut, 1981). 


\section{Pla de Labinas series, Ariège}

$\left(42^{\circ} 38^{\prime} \mathrm{N}, 1^{\circ} 26^{\prime} \mathrm{W}\right)$, alt $+1010 \mathrm{~m}$, Soulcem Valley. Coll and subm 1978 by $\mathrm{G}$ Jalut.

Gif-4805. Palyn 69

Modern

Peaty sediment from 15.5 to $17.5 \mathrm{~cm}$ depth.

Gif-4806. Palyn 70

$330 \pm 90$

Clayey peat from 29.5 to $30.5 \mathrm{~cm}$ depth.

Gif-4807. Palyn 71

$610 \pm 90$

Peat from level 50 to $51 \mathrm{~cm}$ depth.

Gif-4808. Palyn 72

$1430 \pm 90$

Peaty clay from base, 64 to $65 \mathrm{~cm}$ depth.

General Comment (GJ): samples are from levels where pollen was especially abundant. This is explained by erosion due to intensive deforestation at these dates.

\section{Pla de Crouts series, Ariège}

$\left(42^{\circ} 40^{\prime} \mathrm{N}, 1^{\circ} 26^{\prime} \mathrm{W}\right)$, alt $+1850 \mathrm{~m}$. Coll and subm 1978 by G Jalut.

Gif-4809. Palyn 73

$$
500 \pm 90
$$

Sandy peat from 19 to $20 \mathrm{~cm}$ depth.

Gif-4810. Palyn 74

$1960 \pm 100$

Sandy peat from 40 to $41 \mathrm{~cm}$ depth.

Pinet series, Sault, Aude

$\left(42^{\circ} 52^{\prime} \mathrm{N}, 1^{\circ} 58^{\prime} \mathrm{E}\right)$, alt $+880 \mathrm{~m}$. Coll and subm 1972 by G Jalut.

Gif-2446. Palyn 31

$3680 \pm 100$

Peat from 40 to $50 \mathrm{~cm}$ depth. Pollen zone: Sub-boreal.

Gif-2445. Palyn 32

$4530 \pm 110$

Peat from 100 to $110 \mathrm{~cm}$ depth. Pollen zone: Sub-boreal.

Gif-2444. Palyn 33

$7350 \pm 140$

Peat from 290 to $300 \mathrm{~cm}$ depth. Pollen zone: beginning of Atlantic.

Gif-2443. Palyn 34

$8420 \pm 150$ Boreal.

Peat from 400 to $410 \mathrm{~cm}$ depth. Pollen zone: transition Pre-boreal/

Gif-2442. Palyn 35

$9160 \pm 170$

Gray clay from 420 to $430 \mathrm{~cm}$ depth. Pollen zone: Pre-boreal.

General Comment: results agree with expected ages from pollen study. 


\section{Squainfaing series, Vosges}

$\left(48^{\circ} 07^{\prime} \mathrm{N}, 6^{\circ} 59^{\prime} \mathrm{E}\right)$, alt $+850 \mathrm{~m}$. Coll and subm 1975 by M Darmois, Univ Paris IV.

Gif-3622. Squainfaing, $30-50 \mathrm{~cm}$

Modern

Gif-3623. Squainfaing, $250-260 \mathrm{~cm}$

$5280 \pm 130$

Pollen zone: end of Atlantic.

Gif-3624. Squainfaing, $480-500 \mathrm{~cm}$

$2540 \pm 100$

Comment: aberrant result can only be explained by sampling error.

Gif-3723. Squainfaing, $450-460 \mathrm{~cm}$

$6940 \pm 170$

Pollen zone: beginning of Atlantic.

Gif-3653. Col du Surceneux, Vosges

$5030 \pm 140$

$\left(48^{\circ} 06^{\prime} \mathrm{N}, 6^{\circ} 58^{\prime} \mathrm{E}\right)$, alt $+810 \mathrm{~m}$, at depth 360 to $380 \mathrm{~cm}$. Coll and subm 1975 by M Darmois. Pollen zone: beginning of Sub-boreal.

Gif-3872. Le Chargeoir, Vosges

$2120 \pm 100$

Peaty silt from base of bog $\left(48^{\circ} 06^{\prime} \mathrm{N}, 6^{\circ} 53^{\prime} \mathrm{E}\right)$, alt $+895 \mathrm{~m}$, at depth 160 to $180 \mathrm{~cm}$. Coll and subm 1977 by M Darmois.

\section{Gif-3875. Belbriette, Vosges}

$2950 \pm 110$

Peat from base of bog $\left(48^{\circ} 05^{\prime} \mathrm{N}, 6^{\circ} 59^{\prime} \mathrm{E}\right)$, alt $+810 \mathrm{~m}$, at depth 640 to $650 \mathrm{~cm}$. Coll and subm 1977 by M Darmois. Pollen zone: end of Subboreal.

Gif-4938. Saint-Bresson, Haute-Saône

$5300 \pm 300$

Peat from bog, Cirque des Mottots $\left(47^{\circ} 52^{\prime} \mathrm{N}, 6^{\circ} 33^{\prime} \mathrm{E}\right.$ ), alt $+564 \mathrm{~m}$, at depth 155 to $160 \mathrm{~cm}$. Coll and subm 1979 by M Darmois. Pollen zone: end of Atlantic.

\section{Argentina}

Peat from bogs, coll and subm 1973-1975 by V Markgraf, CFR, Gifsur-Yvette, to study postglacial vegetational history in $\mathrm{S}$ hemisphere.

Gif-3656. Atuel, El Sosneado, Prov Mendoza

$3370 \pm 300$

Plant macrofossil layer at depth 558 to $561 \mathrm{~cm}$ in bog $\left(35^{\circ} 10^{\prime} \mathrm{S}, 66^{\circ} 36^{\prime}\right.$ W), alt $+2000 \mathrm{~m}$.

\section{Arroyo Seco series, Cordon del Plata, Prov Mendoza}

$\left(33^{\circ} 10^{\prime} \mathrm{S}, 60^{\circ} 30^{\prime} \mathrm{W}\right)$, alt $+2500 \mathrm{~m}$.

Gif-4318. Arroyo Seco, 7

$820 \pm 90$

Dark soil between two gravelly and sandy levels, at 20 to $35 \mathrm{~cm}$ depth.

Gif-4317. Arroyo Seco, 1

$4090 \pm 110$

Oldest dark soil, at 265 to $280 \mathrm{~cm}$ depth. 
Gif-4316. Cienaga Yalguarez Represa, Uspallata

Organic fibers in gray clay at 100 to $140 \mathrm{~cm}$ depth at base of bog $\left(32^{\circ}\right.$ $10^{\prime} \mathrm{S}, 67^{\circ} \mathrm{W}$ ), alt $+2100 \mathrm{~m}$.

Gif-3657. Mallin Auer, Patagonia

$4390 \pm 300$

Brownish gyttja with clay at 650 to $675 \mathrm{~cm}$ depth at base of bog, $\left(41^{\circ}\right.$ $\left.20^{\prime} \mathrm{S}, 71^{\circ} 42^{\prime} \mathrm{W}\right)$, alt $+1000 \mathrm{~m}$.

Mallin Sonntag series, Patagonia

$\left(41^{\circ} 05^{\prime} \mathrm{S}, 71^{\circ} 33^{\prime} \mathrm{W}\right)$, alt $\mathrm{ca}+800 \mathrm{~m}$.

Gif-3086. Mallin Sonntag 1, 460-485cm depth $\quad 2180 \pm 110$

Gif-3080. Mallin Sonntag 2, 850-875cm depth $\quad 4890 \pm 120$

Gif-3275. Mallin Chileno, Patagonia $\quad \mathbf{8 6 0} \pm 80$

$\left(41^{\circ} 14^{\prime} \mathrm{S}, 71^{\circ} 50^{\prime} \mathrm{W}\right)$, alt ca $+1500 \mathrm{~m}$, at 150 to $155 \mathrm{~cm}$ depth.

Mallin Book series, Patagonia $9.50 \mathrm{~m}$.

Peat from bog $\left(41^{\circ} 20^{\prime} \mathrm{S}, 71^{\circ} 35^{\prime} \mathrm{W}\right)$, alt $+800 \mathrm{~m}$; base of core at

Gif-4035. Mallin Book 1, 185-195cm depth

$$
\begin{aligned}
1600 & \pm 190 \\
6010 & \pm 280 \\
8800 & \pm 170 \\
12,900 & \pm 400
\end{aligned}
$$

Gif-4036. Mallin Book 2, 375-385cm depth

Gif-4037. Mallin Book 3, 550-560cm depth

Gif-3867. Mallin Book 4, 650-655cm depth

General Comment: bog is near lake that was covered with pleniglacial glaciers. Gif-3867 gives min age for retreat of ice.

La Mision series, Tierra del Fuego

$\operatorname{Bog}\left(52^{\circ} 30^{\prime} \mathrm{S}, 67^{\circ} 50^{\prime} \mathrm{W}\right), 3.5 \mathrm{~km}$ inland from present shore.

\section{Gif-3869. La Mision 1}

$8490 \pm 400$

Black clay at 845 to $850 \mathrm{~cm}$ depth.

Gif-3655. La Mision 2

$9300 \pm 180$ depth.

Organic brown layer at 865 to $875 \mathrm{~cm}$ depth: base of core: $900 \mathrm{~cm}$

General Comment: profile is of interest because of its sensitivity to paleoclimatic change. Markgraf (1977) distinguished three zones in pollen diagram. Oldest is almost devoid of tree pollen; principal and longest zone reflects forest expansion, and uppermost zone reflects present treeless steppe. Before forest expansion began, at ca $8000 \mathrm{BP}$, pollen suggests transition period between steppe and forest with more humid climate from 9300 to 8490 BP. 
Gif-3884. Rio Conrintos, Patagonia

$10,100+\begin{array}{r}1200 \\ -750\end{array}$

Uppermost organic varve from varved deposit $\left(43^{\circ} \mathrm{S}, 71^{\circ} 34^{\prime} \mathrm{W}\right)$, alt $+700 \mathrm{~m}$; deposit 10 to $20 \mathrm{~m}$ thick.

\section{Greenland}

Samples from Thule area, NW Greenland. Coll and subm 1972 by J Malaurie, Centre d'Etudes Arctiques, Paris.

\section{Kranak profile, Fjord Murchison}

Peat from bog, $72 \mathrm{~cm}$ thick, $\left(77^{\circ} 43^{\prime} \mathrm{N}, 70^{\circ} \mathrm{W}\right)$.

Gif-3293. Kranak, 5-10cm depth

Gif-3294. Kranak, 25-30cm depth $930 \pm 110$

Gif-2794. Kranak, 30-35cm depth $1020 \pm 100$

\section{Kugkat profile, Fjord Robertson}

Peat from bog, $210 \mathrm{~cm}$ thick, $\left(77^{\circ} 45^{\prime} \mathrm{N}, 70^{\circ} 30^{\prime} \mathrm{W}\right)$.

Gif-2799. Kugkat, 7, 30-35cm depth

$$
\begin{array}{r}
1720 \pm 100 \\
1820 \pm 100 \\
420 \pm 90
\end{array}
$$

Gif-2791. Kugkat, 39, basal level

Gif-2792. Kangerdluarssuk profile, Fjord Bowdoin

Basal level of bog, at $30 \mathrm{~cm}$ depth, $\left(77^{\circ} 43^{\prime} \mathrm{N}, 70^{\circ} \mathrm{W}\right), 5 \mathrm{~km}$ from ice cap.

\section{Atikorlog profile, Fjord Robertson} $\left.30^{\prime} \mathrm{W}\right)$

Organic deposit, $20 \mathrm{~cm}$ thick, overlying sand and gravel, $\left(77^{\circ} 45^{\prime} \mathrm{N}, 70^{\circ}\right.$

Gif-2789. Atikorlog, 8 a, 10-15cm depth

Modern

Gif-2790. Atikorlog, 8 b, 15-20cm depth

Modern

Negri profile, near Neke

Bog, $1 \mathrm{~m}$ thick, $\left(77^{\circ} 45^{\prime} \mathrm{N}, 70^{\circ} 30^{\prime} \mathrm{W}\right)$.

Gif-2971. Negri 2, 5-10cm depth

Modern

Gif-3323. Negri 5, 20-25cm depth

$610 \pm 90$

Gif-2972. Negri 8, 35-40cm depth

$680 \pm 90$

Gif-2973. Negri 9, 40-45cm depth

$710 \pm 90$

Gif-3297. Negri 10, 45-50cm depth

$690 \pm 90$

Gif-3026. Negri 17, 80-85cm depth

$1990 \pm 110$

Gif-2795. Negri 19, 90-95cm depth

$3040 \pm 100$ 
Agparssuit profile, Nakluyt Island

Peat from bog, $30 \mathrm{~cm}$ thick, $\left(77^{\circ} 43^{\prime} \mathrm{N}, 71^{\circ} \mathrm{W}\right)$.

Gif-3298. Agparssuit 2, 3-6cm depth

Gif-3299. Agparssuit 5, 12-15cm depth

Gif-2796. Agparssuit 8-9, 21-27cm depth

Uvdle profile, Fjord Volstenholme

Peat from bog, $2 \mathrm{~m}$ thick, $\left(77^{\circ} 40^{\prime} \mathrm{N}, 69^{\circ} \mathrm{W}\right), 6 \mathrm{~km}$ from ice cap.

Gif-3300. Uvdle 2, 6-12cm depth

$1780 \pm 100$

Gif-3301. Uvdle 6, 30-36 cm depth

$3120 \pm 110$

Gif-3302. Uvdle 13, 72-78cm depth

$3720 \pm 120$

Gif-3303. Uvdle 35, 144-150cm depth

$3540 \pm 120$

Gif-2797. Uvdle 35, 195-200cm depth

$4700 \pm 130$

Saunders profile, Saunders Island

Peat from bog, $56 \mathrm{~cm}$ thick, $\left(77^{\circ} 40^{\prime} \mathrm{N}, 69^{\circ} \mathrm{W}\right)$.

Gif-3959. Saunders 1, 0-6cm depth

$1870 \pm 90$

Gif-3017. Saunders 3, 12-18cm depth

$3380 \pm 100$

Gif-3018. Saunders 5, 24-30cm depth

$3930 \pm 110$

Gif-3019. Saunders 7, 36-42cm depth

$3930 \pm 110$

Gif-2797. Saunders $9,50-56 \mathrm{~cm}$ depth

$4090 \pm 140$

Krassissalik profile, Northemberland Island

Peat from bog, $25 \mathrm{~cm}$ thick, $\left(77^{\circ} 43^{\prime} \mathrm{N}, 71^{\circ} \mathrm{W}\right)$.

Gif-3304. Krassissalik, 0-5cm depth

Modern

Gif-2798. Krassissalik, 20-25cm depth

$1350 \pm 100$

\section{Alaska}

Peat from basal level of coastal bogs. Coll and subm 1974 by J Malaurie.

Gif-3592. Wales, $30-35 \mathrm{~cm}$ depth

$320 \pm 90$

$\left(65^{\circ} 38^{\prime} \mathrm{N}, 168^{\circ} 09^{\prime} \mathrm{W}\right)$

Gif-3593. Shishmaref, $115-120 \mathrm{~cm}$ depth

$1120 \pm 100$

$\left(66^{\circ} 15^{\prime} \mathrm{N}, 166^{\circ} 11^{\prime} \mathrm{W}\right)$ 
Gif-3594. Teller, 60-65cm depth

$\left(65^{\circ} 12^{\prime} \mathrm{N}, 166^{\circ} 23^{\prime} \mathrm{W}\right)$.

Gif-3691. Nome 9, 40-45cm depth

Modern

$\left(64^{\circ} 30^{\prime} \mathrm{N}, 165^{\circ} 30^{\prime} \mathrm{W}\right)$.

Gif-3591. Nome 19, 130-135cm depth

Modern

\section{Glaciers}

France

\section{Gif-2747. Mont-Dore, Massif Central}

Plant branches in clayey lacustrine deposit, behind glacial moraine, $10 \mathrm{~m}$ thick, Vallée de Chaudefour $\left(45^{\circ} 32^{\prime} \mathrm{N}, 2^{\circ} 51^{\prime} \mathrm{E}\right)$, alt $1100 \mathrm{~m}$. Coll and subm 1972 by G Kieffer.

Gif-4128. Santoir Valley R, Cantal

$13,580 \pm 250$

Organic sediment in glacio-fluviatile terrace $\left(45^{\circ} 18^{\prime} \mathrm{N}, 2^{\circ} 47^{\prime} \mathrm{E}\right)$, alt $+900 \mathrm{~m}$. Coll and subm 1977 by Y Veyret, Inst Géog Clermont Ferrand. Comment: dates limits of glaciated land during Tardiglacial in French Massif Central (Veyret, Brousse \& Delibrias, 1978).

\section{Tautal-Haut series, Massif Central}

Wood in morainic deposit, $8 \mathrm{~m}$ thick $\left(45^{\circ} 54^{\prime} \mathrm{N}, 3^{\circ} 07^{\prime} \mathrm{E}\right)$, alt $+910 \mathrm{~m}$. Coll and subm 1982 by R Brousse.

Gif-5891. Tautal-Haut, 3 $\geq 35,000$

Gif-5892. Tautal-Haut, 1 $\geq 35,000$

Gif-5893. Tautal-Haut, 8 $\geq 35,000$

Switzerland

\section{Grundelwald series}

Tree trunks in moraines of glacier $\left(46^{\circ} 38^{\prime} \mathrm{N}, 8^{\circ} 03^{\prime} \mathrm{E}\right)$. Coll and subm 1973 by E Le Roy Ladurie, Coll France, Paris.

Gif-2976. Stieregg, 4 $710 \pm 100$

Alt $1680 \mathrm{~m}$.

Gif-2975. Stieregg, 2

Alt $1680 \mathrm{~m}$.

Gif-2977. Kalli, 6

$1340 \pm 60$

Alt $1650 \mathrm{~m}$. Under Eiger.

Gif-2980. Zasenberg, 10

$910 \pm 60$

Alt $1730 \mathrm{~m}$. 
Gif-3311. Zasenberg, A

$820 \pm 60$

Tree was allegedly dead in AD 1071 according to dendrochonology.

Gif-3312. Zasenberg, B

$1210 \pm 60$

Tree was allegedly dead in AD 1270 according to dendrochronology. General Comment: dates indicate that Grundelwald fossil forest was alive between 7th and 8th centuries at site that is presently entirely denuded. This period corresponds to little climatic optimum of Middle age and of the year, AD 1000 (Delibrias, Le Roy Ladurie, E \& M, 1975).

\section{Chile}

\section{Andes series}

Samples coll and subm 1973-77 by C Laugénie, Univ Pau, to establish chronology of different stages of glacial advances during last glaciation, in S Andes (Laugénie, 1982).

\section{Gif-2614. Rio San Pedro, 4 Ri/sp 2}

$\mathbf{2 7 , 5 0 0} \pm \mathbf{9 4 0}$

Peaty level at top of paleosol, underlain by fluvio-glacial sequence, Pacuno $\left(39^{\circ} 45^{\prime} \mathrm{S}, 72^{\circ} 40^{\prime} \mathrm{N}\right)$.

\section{Gif-2893. Tablaruca cape, Chiloe I, CH/Ta I}

Tree trunk overlying morainic deposit and under neo-glacial terrace, in marine cliff $\left(42^{\circ} 55^{\prime} \mathrm{S}, 73^{\circ} 15^{\prime} \mathrm{W}\right)$.

\section{Gif-2808. Compu, Chiloe I, Level 4}

Tree trunk in peaty level 4 , under fluvial deposit in complex sequence of morainic, fluvio-glacial, and lacustrine deposits, in profile of Compu $\left(42^{\circ}\right.$ $\left.50^{\prime} \mathrm{S}, 72^{\circ} 50^{\prime} \mathrm{W}\right)$.

\section{Gif-2809. Compu, Chiloe I, Level 7}

Plant remains from peaty level 7 , under lacustrine level, in same complex as Gif-2808. Comment: samples Gif-2809 and -2808 correspond to very old glacial fluctuations, as expected.

\section{Gif-2892. Caunahue Valley}

$11,990 \pm 160$

Wood in lacustrine clay, $1 \mathrm{~m}$ above river bed, Caunahue Valley, near estuary in Rancol Lake ( $\left.41^{\circ} 05^{\prime} \mathrm{S}, 72^{\circ} 25^{\prime} \mathrm{W}\right)$. Comment: dates glacial retreat. Age is confirmed by date of other sample coll downstream: 12,200 \pm 400 : Gx-2935 (pers commun).

Gif-3796. Coihueco, Llanquihue

$$
\mathbf{2 4 , 5 0 0} \pm \mathbf{5 0 0}
$$

Peat between two fluvio-glacial sequences, in sec at Rio Coihueco $\left(40^{\circ}\right.$ $50^{\prime} \mathrm{S}, 72^{\circ} 55^{\prime} \mathrm{W}$ ). Comment: dates interstadial of Laguna Bonita.

\section{Gif-3798. Valcahue, Quemchi, Chiloe Island}

$14,200 \pm 160$

Plant remains from thin soil between varved lacustrine silt level overlying moraine at base and fluvio-glacial deposit, at top near Valcahue $\left(42^{\circ} 30^{\prime}\right.$ 
$\left.\mathrm{S}, 72^{\circ} 50^{\prime} \mathrm{W}\right)$. Comment: indicates last glacial readvance began just after $14,200 \mathrm{BP}$.

\section{Los Tacos series, Rinihue}

Samples from sec resulting from seismic crumbling of filling in Los Tacos depression $\left(39^{\circ} 45^{\prime} \mathrm{S}, 72^{\circ} 30^{\prime} \mathrm{W}\right)$.

Gif-2891. Los Tacos, Ri/Tac 3

$28,000 \pm 800$ moraine.

Peat from upper peaty paleosol in alluvial deposit, underlying upper

Gif-3797. Los Tacos, Ri/Tac IV

$29,000 \pm 700$

Plant remains from basal level of same paleosol as Gif-2891.

Gif-2615. Los Tacos, L/Ri/Tac I

$\geq \mathbf{3 4 , 0 0 0}$

Peat from lower peaty paleosol, in alluvial deposit, overlying important lacustrine series which overlies lower moraine.

Gif-2616. Rio Ignao, Ranco L, L/Ra I

Peat from upper part of sequence of alternated deposits of peat and ash, $2.5 \mathrm{~m}$ thick, underlying upper-glacial moraine $\left(40^{\circ} 18^{\prime} \mathrm{S}, 72^{\circ} 37^{\prime} \mathrm{W}\right)$. Comment: age confirmed by Seattle date of tree trunk at top of same level, QL-61: 56,000+2000.

$$
-1700
$$

SEA LEVEL

\section{France}

\section{Jauney Valley series, Lachaize-Giraud, Vendée}

Samples from Holocene fluviatile filling of Jauney Valley $\left(46^{\circ} 41^{\prime} \mathrm{N}, 1^{\circ}\right.$ $47^{\prime}$ W). Coll and subm 1977 by M Ters. Levels are related to present msl.

Gif-4032. Jaunay 10

$1510 \pm 90$

Tree trunk, $0 \mathrm{~m}$.

Gif-4224. Jaunay 8 bis

$1690 \pm 90$

Carbonized wood, $-0.5 \mathrm{~m}$.

Gif-4031. Jaunay 17

$2450 \pm 90$

Carbonized wood, $-1 \mathrm{~m}$.

General Comment: dates only last stage of Holocene filling.

\section{La Mère Valley series, Vendée}

Samples from Holocene filling of La Mère Valley, overlying periglacial grave $\left(46^{\circ} 37^{\prime} \mathrm{N}, 0^{\circ} 46^{\prime} \mathrm{W}\right)$. Coll and subm 1977 by M Ters, Inst Géog, Paris. 
Gif-4226. Mère 2

Carbonized wood in peat.

Gif-4225. Mère 1

Peaty clay.

General Comment: both samples date transition between coarse fluviatile and fine Holocene alluviation.

$$
\text { Italy }
$$

\section{Gif-2746. Punta Penne, Brindisi, DIG 125}

Marine shell debris from deposit of Punta Penne $\left(40^{\circ} 41^{\prime} \mathrm{N}, 17^{\circ} 54^{\prime} \mathrm{E}\right)$, $+1.50 \mathrm{~m}$. Coll 1970 and subm 1972 by I di Geronimo, Inst Geol, Catania, Sicily. Comment: provides recent Holocene age for this deposit usually attributed to Tyrrhenian.

\section{Brazil}

\section{Amazon estuary series}

Bore holes were made down to $50 \mathrm{~m}$ in Quaternary sediments of Marajo Is., Amazon estuary ( $\left.1^{\circ} \mathrm{S}, 48^{\circ} 58^{\prime} \mathrm{W}\right)$. Organic levels subm 1973 by G Siffermann, ORSTOM (Tancredi et al, 1975).

Gif-2962. SE II, 20m depth $\geq 35,000$

Gif-3096. SE II, 12.75-14.10m depth $\geq 35,000$

\section{Venezuela}

\section{Orinoco estuary series}

Samples from cores in Orinoco estuary, coll by E N de Campos and subm 1979 by A Danielo, Nantes.

\section{Gif-2963. Domingo Perez I, A 106}

Peat, at base of peaty level, 200 to $300 \mathrm{~cm}$ depth, Profile A $106\left(8^{\circ} 33^{\prime}\right.$ $\left.\mathrm{N}, 61^{\circ} 05^{\prime} \mathrm{W}\right)$.

\section{Gif-2964. Cano Guinamorena, B 257}

Peat from peaty level, $-2 \mathrm{~m}, 340$ to $400 \mathrm{~cm}$ depth, Profile B $257\left(9^{\circ} 45^{\prime}\right.$ $\left.\mathrm{N}, 62^{\circ} 15^{\prime} \mathrm{W}\right), 30 \mathrm{~km}$ from present coast.

General Comment: dates recent sedimentation in estuary, sedimentation related to Flandrian tansgression mainly for B 257.

\section{Greenland}

\section{Thule Kranak profile series}

Shell samples from uplift levels on left bank of Nagssuak Kuk R, $3 \mathrm{~km}$ from estuary $\left(77^{\circ} 43^{\prime} \mathrm{N}, 70^{\circ} \mathrm{W}\right)$.

Gif-3020. Kranak, $+50 \mathrm{~m}$

$8260 \pm 150$ 
Gif-3021. Kranak, $+40 \mathrm{~m}$

Gif-3305. Kranak, $+25-30 \mathrm{~m}$

Gif-3022. Kranak, $+15 \mathrm{~m}$

Shell from Siorapaluk $\left(77^{\circ} 48^{\prime} \mathrm{N}, 70^{\circ} 58^{\prime} \mathrm{W}\right),+60 \mathrm{~m}$.

Gif-3212. Ivnartalik

$7600 \pm 400$

Shell from Ivnartalik $\left(77^{\circ} 50^{\prime} \mathrm{N}, 71^{\circ} \mathrm{W}\right)$.

VOLCANOES

France

Massif Central

Gif-3590. Puy de Mey, 8

$1760 \pm 90$

Paleosol III, Puy de Mey (45 43' N, $\left.2^{\circ} 56^{\prime} \mathrm{E}\right)$. Coll and subm 1975 by R Brousse, Fac Sci, Orsay.

Gif-6229. Bois de Ceyssat

$3490 \pm 80$

Wood in paleosol under pyroclastic deposit, $1 \mathrm{~m}$ thick, Bois de Ceyssat $\left(45^{\circ} 45^{\prime} \mathrm{N}, 2^{\circ} 59^{\prime} \mathrm{E}\right)$. Coll and subm 1983 by R Brousse.

Gif-3589. Pont de Rivallet, 2

$6900 \pm 500$

Paleosol under Tartaret flow, near Pont de Rivallet $\left(45^{\circ} 35^{\prime} \mathrm{N}, 3^{\circ} 03^{\prime}\right.$ E). Coll and subm 1975 by R Maury, Fac Sci, Orsay.

\section{Gif-6152. Puy de Cliziaux}

$7750 \pm 90$

Wood in paleosol under black lapilli deposit, $40 \mathrm{~cm}$ thick, at Puy de Cliziaux $\left(45^{\circ} 08^{\prime} \mathrm{N}, 3^{\circ} \mathrm{E}\right)$, Chaîne des Puys. Coll and subm 1983 by R Brousse.

\section{Gif-6231. Cheire de Mercoeur}

$8110 \pm 90$

Charcoal at base of trachyandesitic ash level overlain by basaltic ash, Cheir de Mercoeur, Chaine des Puys ( $\left.45^{\circ} 43^{\prime} \mathrm{N}, 2^{\circ} 56^{\prime} \mathrm{E}\right)$, alt $870 \mathrm{~m}$. Coll and subm 1983 by R Brousse.

Gif-6228. Puy de Tenusset

$8150 \pm 380$

Wood, Puy de Tenusset, Chaîne des Puys ( $\left.45^{\circ} 52^{\prime} \mathrm{N}, 2^{\circ} 56^{\prime} \mathrm{E}\right)$, alt $960 \mathrm{~m}$. Coll and subm 1983 by R Brousse.

Gif-6230. Puy du Pourcharet

Charcoal in scoria level, Puy du Pourcharet, Chaîne des Puys ( $45^{\circ} 43^{\prime}$ $\mathrm{N}, 2^{\circ} 56^{\prime} \mathrm{E}$ ) alt $1060 \mathrm{~m}$. Coll and subm 1983 by R Brousse. 
Charcoal in volcanic scoria levels, plain between Puy de Louchadière and Puy Chopine $\left(45^{\circ} 50^{\prime} \mathrm{N}, 2^{\circ} 56^{\prime} \mathrm{E}\right)$, Chaîne des Puys. Coll and subm 1975 by H Pelletier, Fac Sci, Clermont-Ferrand. Comment: dates trachyandesitic eruption of Puy Chopine.

Gif-5133. Jaujac

$15,000 \pm 360$ rais.

Charcoal under ash from Jaujac volcano $\left(44^{\circ} 38^{\prime} \mathrm{N}, 4^{\circ} 15^{\prime} \mathrm{E}\right)$, Viva-

\section{Gif-5132. Monpezat}

$16,280 \pm 420$

Charcoal under Monpezat basaltic flow, Château de Pourcheyrolles, Vivarais $\left(44^{\circ} 43^{\prime} \mathrm{N}, 4^{\circ} 22^{\prime} \mathrm{E}\right)$. Coll and subm 1980 by R Brousse.

Gif-2640. Pont de Labeaume, Vals

$11,770 \pm 270$

Paleosol under Pont de Labeaume flow ( $\left.44^{\circ} 39^{\prime} \mathrm{N}, 4^{\circ} 16^{\prime} \mathrm{E}\right)$, Ardèche. Coll and subm 1972 by R Maury and R Brousse.

\section{Gif-2642. Egaules}

Wood under lava flow, Egaules ( $45^{\circ} 50^{\prime} \mathrm{N}, 2^{\circ} 59^{\prime} \mathrm{E}$ ), Chaine des Puys. Coll and subm 1972 by R Brousse.

Gif-2643. Souilhols, 535 e

Wood under scoria from Souilhols volcano $\left(44^{\circ} 40^{\prime} \mathrm{N}, 4^{\circ} 15^{\prime} \mathrm{E}\right)$, Ardèche. Coll and subm 1972 by R Maury and R Brousse.

\section{Olby series}

Two lava flows with reversed remanent magnetism were discovered 1967 at Laschamp and Olby $\left(45^{\circ} 44^{\prime} \mathrm{N}, 2^{\circ} 51^{\prime} \mathrm{E}\right)$, in Chaîne des Puys, by N Bonhommet and $\mathrm{J}$ Balkine. This event was thought to have occurred between 20,000 and $8000 \mathrm{yr}$ ago (Bonhommet \& Zahringer, 1969). New ${ }^{14} \mathrm{C}$, TL, and K-Ar dates at "Les Plats, Olby" indicate much greater age values (Gillot et al, 1979).

\section{Gif-4007. Olby 1}

$$
\mathbf{2 6 , 8 0 0} \pm \mathbf{8 0 0}
$$

Residual organic fraction after extraction of humic acids from soil under Olby lava flow. Coll 1977 by J Labeyrie. Comment: insufficient humic acids dating.

\section{Gif-4563. Olby 2}

Humic fraction, extracted at pH 9.87 from 2nd soil sample, coll 1978 at same loc as Gif-4007.

Gif-4564. Olby 3 4563.

Humic fraction, extracted at $\mathrm{pH} 11.5-12$, from same soil as Gif- 
Gif-4565. Olby 4

Residual fraction, after extraction of humic fraction, from same soil as Gif-4563.

General Comment: ${ }^{14} \mathrm{C}$ age of Olby organic layer is $\geq 36,000 \mathrm{yr}$ : it agrees with TL and K-Ar ages obtained for lava flow, which are 36,000 \pm 4000 and $42,000 \pm 5000$, respectively.

\section{Italy}

\section{Vesuvius series}

Samples dated to establish eruptive history of Somma-Vesuvius volcanic complex from study of pyroclastic sequences of Monte Somma $\left(40^{\circ}\right.$ $49^{\prime} \mathrm{N}, 14^{\circ} 26^{\prime} \mathrm{E}$ ) in order to evaluate volcanic risks in Naples region (Delibrias et al, 1979). Coll and subm 1977-79 by D Paola, CNR, Pisa, R Santacroce and G Marinelli, Univ Pisa.

Gif-5266. Cava "La Marca”, PFSV 308 $470 \pm 60$

Charcoal in paleosol, lying on ash.

Gif-5265. Cava "La Marca", PFSV 306 $1550 \pm 60$

Paleosol, under ash and paleosol Gif-5266; sequence above historic pumice level of $\mathrm{AD} 472$.

Gif-5268. Cava unova tra Pollena e Cercola, PFSV $315 \quad 330 \pm 60$

Charcoal in pumice supposed to be Pompei level. Comment: date does not confirm hypothesis.

Gif-4203. Cava Lagno Amendolare, I $1030 \pm 90$

Wood under nuée ardente deposit. Coll and subm by G Marinelli.

Gif-4205. Cava Lagno Amendolare, PFSV $86 \quad 1750 \pm 90$

Charcoal in nuée ardente deposit, $1 \mathrm{~m}$ thick, above pumice supposedly corresponding to Pompei eruption, AD 79. Comment: date supports hypothesis.

Gif-4206. Lagno di Pollena, PFSV 83 $1600 \pm 60$ Pompei.

Carbonized tree trunk in nuée ardente deposit, above pumice level of

Gif-4250. Cava Lagno Amendolare, PFSV 86 bis $\quad 1590 \pm 60$

Carbonized wood in nuée ardente deposit above Pompei level.

Gif-4483. Lagno di Pollena, PFSV 101

$1280 \pm 50$

Wood in lahar above nuée ardente deposit.

Gif-5098. Irpina, 3

$1890 \pm 60$

Carbonized wood in unit dated to Pompei level $\left(40^{\circ} 45^{\prime} \mathrm{N}, 14^{\circ} 27^{\prime} \mathrm{E}\right)$. Coll and subm by G Camus, Fac Sci, Clermont-Ferrand. 
Gif-5097. Ottaviano, 2

Carbonized wood in soil from sequence above pumice level of Avellino $\left(40^{\circ} 51^{\prime} \mathrm{N}, 14^{\circ} 29^{\prime} \mathrm{E}\right)$. Coll and subm by $\mathrm{G}$ Camus.

Gif-4484. Cava Primavera, PFSV 105

$1800 \pm 60$

Wood in lahar between nuée ardente deposit and Pompei level.

Gif-4377. Cava Primavera, PFSV 104

$1810 \pm 50$

Carbonized wood in pumice of Pompei level, quarry 2.

Gif-5264. Cava Primavera, PFSV 300

$3530 \pm 70$

Paleosol on ashy layer between pumices of Pompei and Avellino dated by Gif-4517 (below). Coll and subm by R Santacroce.

Gif-5096. Ottaviano, 1

$3170 \pm 70$

Carbonized wood in soil above pumice of Avellino. Coll and subm 1979 by $\mathrm{G}$ Camus.

Gif-4486. Terzigno, PFSV 156

$3600 \pm 80$

Paleosol underlying pumice.

Gif-4517. Astroni, PFSV 210

$3760 \pm 70$

Paleosol under Avellino pumice.

Gif-5267. Cava Loop, PFSV 314

$2750 \pm 200$

Paleosol in pumice supposedly Pompei or Avellino level. Comment: date does not confirm hypothesis.

Gif-4485. Santa Teresella, PFSV 115

$5530 \pm 80$

Paleosol underlying pumice of Astroni.

Gif-5262. Cava Lagno Amendolare, PFSV $295 \quad 6870 \pm 130$

Paleosol under nuée ardente deposit. Comment: unrelated to Pompei event, as expected.

Gif-4378. Case Traianello, PFSV 112

$7910 \pm 100$

Upper part of paleosol overlying pumice of Agnano and underlying "Pomici Gemelli."

Gif-4379. Case Traianello, PFSV 113

$8470 \pm 100$

Lower part of same paleosol as Gif-4378.

Gif-5099. Avellino, 4

$8110 \pm 100$

Carbonized wood in unit under Avellino pumice $\left(40^{\circ} 49^{\prime} \mathrm{N}, 14^{\circ} 47^{\prime} \mathrm{E}\right)$. Coll and subm 1979 by G Camus. 
Gif-4251. Cava Lagno Amendolare, PFSV 89

$8830 \pm 70$

Paleosol, same strat position as paleosol Gif-4378. Humic acids from same sample were dated, $8650 \pm 130$. Comment: these paleosols do not seem contaminated and thus give significant ages.

Gif-4376. Cava Lagno Amendolare, PFSV 111

$9760 \pm 300$

Paleosol underlain by pumice of Agnano.

Gif-4488. Cava Primavera, PFSV 110

$11,400 \pm 130$

Paleosol between two pumices dated at Cava Amendolare by Gif-4376 and -4375 .

Gif-4487. Cava Primavera, PFSV 109

$14,120 \pm 160$

Paleosol between two pyroclastic units above pumice of Amendolare dated by Gif- 4375 .

Gif-4375. Cava Lagno Amendolare, PFSV $106 \quad 17,050 \pm 140$

Paleosol on pyroclastic unit "Pomici di base" overlying lava from Monte Somma.

General Comment: Pompei level, well dated, has been important datum to establish stratigraphy of recent eruptions of Vesuvius.

\section{Etna series}

Samples from Etna $\left(37^{\circ} 42^{\prime} \mathrm{N}, 15^{\circ} 02^{\prime} \mathrm{E}\right)$. Coll and subm $1972-1974$ by $\mathrm{G}$ Kieffer, Inst Geog, Clermont-Ferrand, to date some important eruptions of last eruptive sequence (Kieffer, 1979, 1985).

Gif-3070. Etna, 74 II

$1810 \pm 90$

Carbonized wood in pumiceous ash, W side of Etna, alt $1980 \mathrm{~m}$. Comment: dates very important eruption.

\section{Gif-3071. Etna, 74 III}

$1760 \pm 90$

Tree trunk in ash, NE side of Etna, alt $1980 \mathrm{~m}$. Comment: dates very important eruption.

\section{Gif-2775. Etna, 732}

$1320 \pm 90$

Wood in fine ash, overlying lapilli layer, S side of Etna $\left(37^{\circ} 42^{\prime} \mathrm{N}, 15^{\circ}\right.$ $01^{\prime} \mathrm{E}$ ), alt $1600 \mathrm{~m}$. Comment: corresponds to explosive episode.

Gif-2776. Etna, 73 V

$3230 \pm 110$

Carbonized tree trunk in fine ash, overlying brown-colored pumice, on $\mathrm{S}$ side of Etna, alt $1600 \mathrm{~m}$. Comment: corresponds to explosive episode.

Gif-2777. Etna, 73 VI

$6100 \pm 140$

Carbonized wood in brown pumice overlying thin yellow ash on ancient lava flows, S side of Etna. Comment: Gif-2775, -2776, -2777 are superimposed and correspond to three explosive episodes. 
Gif-3428. Etna, 74 V

$2840 \pm 110$

Carbonized wood under andesitic ash, some $m$ thick, Zafferrana-Rifugio Sapienza $\operatorname{Rd}\left(37^{\circ} 41^{\prime} \mathrm{N}, 2^{\circ} 36^{\prime} \mathrm{E}\right)$, alt $1600 \mathrm{~m}$.

Gif-2448. Etna, 722

$1840 \pm 90$

Branches of Pinus laricio in reworked pumice deposit on caldera lip, SW side of Etna, alt $1750 \mathrm{~m}$.

Gif-2778. Etna, 73 IX

$4280 \pm 110$

Carbonized wood in fine ash NW side of Etna, alt $1800 \mathrm{~m}$. Comment: corresponds to explosive episode.

Gif-3427. Etna 74 IV

$5460 \pm 130$

Wood in ash, at bottom of cliff of "Olmo," near Torre Archirafi, $2 \mathrm{~m}$ above msl. Comment: dates beginning of last stage of formation of Valle del Bove.

Gif-3429. Etna 74 VI

$8140 \pm 190$

Carbonized wood under andesitic ash, Cassone-Monte Pomociaro $\operatorname{wood}\left(37^{\circ} 41^{\prime} \mathrm{N}, 2^{\circ} 36^{\prime} \mathrm{E}\right)$, alt $1400 \mathrm{~m}$. Comment: dates one of oldest explosive eruptions of last sequence of acid eruptions of Etna.

Gif-4618. Etna, 78 I

$14,180 \pm 260$

Carbonized tree trunk under pyroclastic flow, NE Biancavilla, alt $+590 \mathrm{~m}$. Coll and subm 1978 by G Kieffer.

\section{Gif-3069. Etna, 74 I}

$18,100 \pm 400$

Soil under flow lava at base of $\mathrm{W}$ hillside of Etna.

\section{Lipari Is. series}

Samples from pyroclastic surge deposits of Monte Guardia, S Lipari Is. $\left(38^{\circ} 28^{\prime} \mathrm{N}, 14^{\circ} 58^{\prime} \mathrm{E}\right)$. Deposits represent sequences III and IV of volcanic activity. Coll and subm 1980-81 by G Zuffa and R Mazzuoli, Univ Calabria, Consenza (Crisci et al, 1983).

Gif-5326. Lipari, sec VII, LR 61

Organic horizon from upper ash-flow units.

Gif-5371. Lipari, sec VII, LR 60

Organic horizon from upper ash-flow units.

Gif-5591. Lipari, sec I, LR 88

$20,300 \pm 700$

Organic horizon, poor in organic carbon, from upper ash-flow units. Comment: undersized sample.

Gif-5328. Lipari, sec I, LR 66

Carbonaceous earth, sec I, overlying andesitic unit designed as "Key bed," overlain by surge deposits of Monte Guardia. 
Gif-5587. Lipari, sec I, LR 84

$22,480 \pm 1100$

Organic horizon, poor in organic carbon, underlying "key bed."

Gif-5375. Lipari, sec I, LR 68

$23,500 \pm 900$

Organic horizon, underlying Gif-5587.

Gif-5327. Lipari, sec XVIII, LR 64

Wood, in unit above deposits of Monte San Angelo eruption, at base of lower ash-flow units.

\section{Lesser Antilles}

\section{Guadeloupe}

\section{La Soufrière series}

Wood and charcoal dated to establish chronology for volcanism of La Soufrière. This study was made by Paterne (1980).

Gif-3014. Quarry of Pintade, Basse Terre I

Charcoal in pumice $\left(16^{\circ} 00^{\prime} \mathrm{N}, 61^{\circ} 44^{\prime} \mathrm{W}\right)$. Coll and subm 1973 by $\mathrm{M}$ Feuillard, St Claude, Guadeloupe.

Gif-3015. Quarry of Pintade, Basse Terre 2

$14,950 \pm 200$

Charcoal in pumice. Coll and subm 1973 by M Feuillard. Comment: aberrant date can only be explained by sampling error.

Gif-3031. Quarry of Pintade, Basse Terre 3

Charcoal in pumice. Coll and subm by M Feuillard.

Gif-4729. Quarry Danois, GB 42

Charcoal in rose-colored pumice flow of Pintade. Coll by J Dagain and subm 1979 by D Westercamp, BRGM, Orléans.

Gif-4732. Rivière du Pérou, GB 45

$\geq \mathbf{3 8 , 5 0 0}$

Charcoal in lahar of phreatic origin.

Gif-4725. Rivière du Galion, GB 38

Carbonized wood in yellowish lahar $\left(16^{\circ} 24^{\prime} \mathrm{N}, 61^{\circ} 41^{\prime} \mathrm{W}\right)$. Coll by $\mathrm{J}$ Dagain and subm 1979 by D Westercamp.

Gif-4343. Ravine Chaude, GB 6

Wood in paleosol, under gray clay unit, lying on cinerite deposit $\left(16^{\circ}\right.$ $01^{\prime} \mathrm{N}, 61^{\circ} 40^{\prime} \mathrm{W}$ ), alt $700 \mathrm{~m}$. Coll and subm 1977 by D Westercamp.

Gif-4344. Ravine Chaude, GB 8 $\geq 37,000$

Carbonaceous soil under cinerite layer.

Gif-4703. Ravine Chaude, GB 7 $\geq \mathbf{3 8 , 5 0 0}$

Charcoal, $1.8 \mathrm{~m}$ under Gif-4344. Coll and subm 1977 by D Westercamp. 
Gif-4707. Mouth of Grand Carbet Riv, GB 17

Humic acid from carbonaceous remains in flow of white and gray pumice with gray ash. Coll and subm 1978 by D Westercamp.

Gif-4709. Mouth of Grand Carbet Riv, GB 21

Charcoal in gray ash $\left(16^{\circ} 01^{\prime} \mathrm{N}, 61^{\circ} 34^{\prime} \mathrm{W}\right)$. Coll and subm 1978 by D Westercamp.

Gif-4708. Mouth of Grand Carbet Riv, GB 20

$24,300 \pm 900$

Charcoal fragments in nuée ardente deposit of fine gray ash with lapilli, $1 \mathrm{~m}$ above Gif-4709. Coll and subm 1978 by D Westercamp.

Gif-3032. Mouth of Grand Carbet Riv

$21,340 \pm 400$

Charcoal from ash layer, $0.6 \mathrm{~m}$ thick, lying on pumice and underlying nuée ardente deposit, $7 \mathrm{~m}$ thick. Coll and subm 1973 by M Feuillard.

Gif-3016. Anse à la Fontaine, Mouth of Grand Carbet Riv

$\mathbf{2 5 , 5 0 0} \pm \mathbf{5 0 0}$

Charcoal fragments in nuée ardente lying on pumice deposit. Coll and subm 1973 by M Feuillard.

Gif-4346. Rivière du Grand Carbet

$29,800 \pm 800$

Charcoal in nuée ardente deposit of Saint-Vincent type underlying Gif-3016 and -3032. Coll and subm 1977 by D Westercamp.

Gif-4715. Rivière du Grand Carbet, GB 27

$23,800 \pm 500$

Charcoal in deposit of hot lahar originating in nuée ardente of porphyric andesite, alt $120 \mathrm{~m}$. Coll and subm 1978 by J Dagain and D Westercamp.

Gif-4721. Rivière Grande Anse, GB 34

$23,200 \pm 600$

Wood in reworked fm with abundant light porphyric pumice $\left(15^{\circ} 59^{\prime}\right.$ $\left.\mathrm{N}, 61^{\circ} 39^{\prime} \mathrm{W}\right)$, alt $60 \mathrm{~m}$. Coll and subm 1978 by J Dagain and D Westercamp.

Gif-4717. Rivière du Grand Carbet, GB 30

$21,730 \pm 550$

Fine charcoal fragments in soil of ashy nuée ardente deposit of porphyric andesite, on right bank of Rivière du Grand Carbet $\left(16^{\circ} 02^{\prime} \mathrm{N}, 61^{\circ}\right.$ $36^{\prime} \mathrm{W}$ ), alt $240 \mathrm{~m}$. Coll and subm 1978 by J Dagain and D Westercamp.

\section{Gif-4718. Rivière du Grand Carbet, GB 31}

$23,450 \pm 600$

Wood in lahar of phreatic origin, under nuée ardente deposit containing charcoal dated by Gif-4717. Coll and subm 1978 by J Dagain and D Westercamp.

Gif-4714. Rivière du Grand Carbet, niv 4

$$
24,200 \pm 600
$$

Charcoal fragments in nuée ardente deposit of dark gray porphyric andesite, $5 \mathrm{~m}$ thick, alt $105 \mathrm{~m}$. Coll and subm 1978 by J Dagain and D Westercamp. 
Gif-4347. Rivière du Grand Carbet, niv 2

$17,800 \pm 400$

Charcoal fragments in deposit of nuée ardente composed of dark gray andesite, above nuée ardente deposit of Saint Vincent type dated by Gif4714. Coll and subm 1977 by D Westercamp.

Gif-4719. Morne Dongo, GB 32

$13,800 \pm 260$

Carbonaceous soils alternating with coarse ash units of variable grain size $\left(16^{\circ} 02^{\prime} \mathrm{N}, 61^{\circ} 37^{\prime} \mathrm{W}\right)$, alt $290 \mathrm{~m}$. Coll and subm 1978 by J Dagain and D Westercamp.

Gif-4720. Morne Dongo, GB 33

$14,500 \pm 200$

Yellowish-brown clay under Gif-4719.

Gif-4345. Rivière du Galion, 7

$12,700 \pm 230$

Wood in volcanic-sedimentary sequence with vitreous pyroclastic rocks, $4 \mathrm{~m}$ thick $\left(16^{\circ} 21^{\prime} \mathrm{N}, 61^{\circ} 39^{\prime} \mathrm{W}\right)$, alt $670 \mathrm{~m}$. Coll and subm by D Westercamp.

Gif-4706. Rivière du Galion, 5, GB II

$13,640 \pm 250$

Wood in lahar of phreatic eruption. Coll and subm 1978 by D Westercamp.

Gif-4724. Rivière du Galion, GB 37

$8500 \pm 100$

Wood in lahar of phreatic origin $\left(16^{\circ} 00^{\prime} \mathrm{N}, 61^{\circ} 42^{\prime} \mathrm{W}\right)$, alt $100 \mathrm{~m}$. Coll and subm 1978 by J Dagain and D Westercamp.

Gif-4723. Rivière du Galion, GB 36

$7700 \pm 140$

Wood in lahar with dark gray andesite blocks, alt $90 \mathrm{~m}$. Coll and subm 1978 by J Dagain and D Westercamp.

Gif-4713. Morne Dolé, GB 25

$4600 \pm 80$

Carbonized wood in the upper part of the nuée ardente sequence containing lapilli of bicolored andesite $\left(16^{\circ} 00^{\prime} \mathrm{N}, 61^{\circ} 40^{\prime} \mathrm{W}\right)$, alt $908 \mathrm{~m}$. Coll and subm 1978 by J Dagain and D Westercamp.

Gif-4712. Morne Dolé, GB 24

$4400 \pm 110$

Carbonized wood in yellowish-brown clay overlying Gif-4713.

Gif-4730. Rivière Grande Anse, GB43

$3600 \pm 60$

Wood in lahar of phreatic origin $\left(16^{\circ} 30^{\prime} \mathrm{N}, 61^{\circ} 39^{\prime} \mathrm{W}\right)$, alt $500 \mathrm{~m}$. Coll and subm 1978 by J Dagain and D Westercamp.

Gif-4700. Rivière Grande Anse, GB 2

$3500 \pm 90$

Fragments of wood in lahar composed of reworked pyroclastic deposits $\left(16^{\circ} 00^{\prime} \mathrm{N}, 61^{\circ} 39^{\prime} \mathrm{W}\right)$, alt $640 \mathrm{~m}$. Coll and subm 1978 by D Westercamp. 
Gif-3035. Rivière du Galion, Morin site

$3450 \pm 100$

Wood from lower part of sequence of superimposed lahars, $50 \mathrm{~m}$ thick, alt $210 \mathrm{~m}$. Coll and subm 1973 by M Feuillard.

Gif-4342. Morne Savon, Matouba

$3200 \pm 100$

Carbonized wood in nuée ardente deposit of pale violet andesite, $1 \mathrm{~m}$ thick $\left(16^{\circ} 20^{\prime} \mathrm{N}, 61^{\circ} 42^{\prime} \mathrm{W}\right)$, alt $518 \mathrm{~m}$. Coll and subm 1977 by D Westercamp.

Gif-4710. Rivière aux Herbes, GB 22

$3200 \pm 100$

Wood from upper part of sequence of superimposed lahars, with and without wood $\left(16^{\circ} \mathrm{N}, 61^{\circ} 43^{\prime} \mathrm{W}\right)$, alt $180 \mathrm{~m}$. Coll and subm 1978 by $\mathrm{J}$ Dagain.

Gif-4716. Rivière du Grand Carbet, GB 28

$2850 \pm 70$

Wood from lahar of phreatic origin $\left(16^{\circ} 02^{\prime} \mathrm{N}, 61^{\circ} 36^{\prime} \mathrm{W}\right)$, alt $230 \mathrm{~m}$. Coll and subm 1978 by J Dagain and D Westercamp.

Gif-3034. Morne Dolé

$2800 \pm 100$

Wood in lahar of reworked nuée ardente deposit, alt $340 \mathrm{~m}$. Coll and subm 1973 by M Feuillard.

Gif-4731. Rivière du Galion, GB 44

$2700 \pm 80$

Wood in lahar of phreatic origin, alt $310 \mathrm{~m}$. Coll and subm 1979 by J Dagain and D Westercamp.

Gif-4705. Rivière aux Herbes, GB 10

$2550 \pm 70$

Wood in lahar with abundant light andesite blocks. Coll and subm 1978 by D Westercamp.

Gif-4702. Ravine Chaude, GB 5

$1700 \pm 100$

Wood in lahar from phreatic eruption $\left(16^{\circ} 02^{\prime} \mathrm{N}, 61^{\circ} 40^{\prime} \mathrm{W}\right)$. Coll and subm 1978 by $D$ Westercamp.

Gif-4734. Valkanaers, near Gourbeyre, GB 50

$1370 \pm 100$

Peat, alt $300 \mathrm{~m}$.

Gif-4735. Rivière du Galion, GB 47 A

$680 \pm 60$

Wood from level with carbonized tree trunks in reworked pumice and black cinders, alt $570 \mathrm{~m}$. Coll and subm 1979 by J Dagain and D Westercamp.

Gif-4736. Rivière du Galion, GB 47 B

$690 \pm 90$

Similar to Gif-4735. Coll and subm 1979 by M Paterne.

Gif-4737. Rivière du Galion, GB 48

$650 \pm 50$

Similar to Gif-4735. Coll and subm 1979 by M Paterne. 
Gif-4738. Savane à Mulets, GB 49

Carbonized wood in pumice overlying violet nuée ardente deposit, Gif$4704\left(16^{\circ} 02^{\prime} \mathrm{N}, 61^{\circ} 40^{\prime} \mathrm{W}\right)$, alt $1050 \mathrm{~m}$. Coll and subm 1979 by J Dagain and D Westercamp.

Gif-4704. Ravine de la Matyles, GB 9

$460 \pm 90$

Wood in lahar from phreatic eruption, under Gif-4738 $\left(16^{\circ} 02^{\prime} \mathrm{N}, 61^{\circ}\right.$ $39^{\prime} \mathrm{W}$ ), alt $1130 \mathrm{~m}$. Coll and subm 1978 by D Westercamp.

Gif-4733. Trois Rivières, GB 46

$\mathbf{2 0 0} \pm \mathbf{5 0}$

Charcoal in clay, lying on andesite flows of Trois Rivières $\left(16^{\circ} 58^{\prime} \mathrm{N}\right.$, $61^{\circ} 38^{\prime} \mathrm{W}$ ), alt $300 \mathrm{~m}$. Coll and subm 1979 by J Dagain and D Westercamp.

Gif-3033. Savane à Mulets

$200 \pm 80$

Wood in non-indurated pumice, $1 \mathrm{~m}$ thick, overlying volcanic sand, alt 1130m. Coll and subm 1973 by M Feuillard.

Gif-4722. Rivière Grande Anse, GB 35

Wood in rock fall $\left(16^{\circ} 25^{\prime} \mathrm{N}, 61^{\circ} 39^{\prime} \mathrm{W}\right)$, alt $490 \mathrm{~m}$. Coll and subm 1978 by J Dagain and D Westercamp.

\section{Martinique}

\section{Montagne Pelée series}

Samples dated to establish chronology of volcanic eruptions of Montagne Pelée.

Gif-4752. Junction of Rivières Capot and Daniel, MPB 72

Carbonized tree trunks in gray ash underlying pyroclastic deposit overlain by nuée ardente $\left(14^{\circ} 16^{\prime} \mathrm{N}, 61^{\circ} 07^{\prime} \mathrm{W}\right)$, alt $320 \mathrm{~m}$.

\section{Gif-4753. Junction of Rivières Capot and Daniel,} MPB 73

Carbonized wood in nuée ardente deposit, under Gif-4752.

Gif-4760. Rivière Providence, MPB 90 $\geq \mathbf{4 0 , 0 0 0}$

Carbonized wood in weathered nuée ardente deposit $\left(14^{\circ} 47^{\prime} \mathrm{N}, 61^{\circ}\right.$ $07^{\prime} \mathrm{W}$ ), alt $330 \mathrm{~m}$. Coll and subm 1979 by D Westercamp.

Gif-4761. Ravine Abd el Kader, MPB 91

$26,500 \pm 600$

Carbonized wood from lower part of pyroclastic flow unit with weathered vitreous bicolored cinders $\left(14^{\circ} 48^{\prime} \mathrm{N}, 61^{\circ} 07^{\prime} \mathrm{W}\right)$, alt $380 \mathrm{~m}$. Coll and subm 1979 by D Westercamp.

Gif-4765. Rivière La Falaise, MPB 95

Carbonized large pieces of wood in ashy nuée ardente deposit. $\left(14^{\circ} 49^{\prime}\right.$ $\mathrm{N}, 61^{\circ} 08^{\prime} \mathrm{W}$ ), alt $290 \mathrm{~m}$. Coll and subm 1979 by D Westercamp. 
Gif-4764. Rivière La Falaise, MPB 94

Carbonized wood in weathered blackish pumice and scoria $\left(14^{\circ} 49^{\prime} \mathrm{N}\right.$, $61^{\circ} 08^{\prime} \mathrm{W}$ ), alt $300 \mathrm{~m}$. Coll and subm 1979 by D Westercamp.

\section{Gif-4759. Road from Fond Labour to Morne Degras,} MPB 79

Abundant carbonized wood in nuée ardente deposit $\left(14^{\circ} 48^{\prime} \mathrm{N}, 61^{\circ} 06^{\prime}\right.$ W), alt $170 \mathrm{~m}$. Coll and subm 1977 by D Westercamp.

Gif-4767. Ravine from Trou Congo to Vivies, MPB 97

$10,280 \pm 180$

Carbonized wood in white pumice flow unit $\left(14^{\circ} 49^{\prime} \mathrm{N}, 6 \mathrm{i}^{\circ} 06^{\prime} \mathrm{W}\right)$, alt $120 \mathrm{~m}$. Coll and subm 1979 by D Westercamp.

Gif-4782. Rivière des Pères, MPB 32

$6630 \pm 130$

Carbonized wood in pumice flow deposit $\left(14^{\circ} 16^{\prime} \mathrm{N}, 61^{\circ} 10^{\prime} \mathrm{W}\right)$, alt $190 \mathrm{~m}$. Coll and subm 1973 by D Westercamp.

Gif-4768. Rivière La Falaise, MPB 98

$5070 \pm 110$

Carbonized wood from lower part of black scoria flow, $4 \mathrm{~m}$ thick, overlain by important nuée ardente deposit, Merapi type $\left(14^{\circ} 49^{\prime} \mathrm{N}, 61^{\circ} 08^{\prime} \mathrm{W}\right)$, alt $440 \mathrm{~m}$. Coll and subm 1979 by D Westercamp.

Gif-4751. Rivière Marie Luce, MPB 71

$4780 \pm 80$

Wood in lahar $\left(14^{\circ} 46^{\prime} \mathrm{N}, 61^{\circ} 07^{\prime} \mathrm{W}\right)$, alt $355 \mathrm{~m}$. Coll and subm 1977 by D Westercamp.

Gif-4763. Rivière La Falaise, MPB 93

$4710 \pm 70$

Carbonized wood in nuée ardente deposit with abundant blocks (14 ${ }^{\circ}$ $49^{\prime} \mathrm{N}, 61^{\circ} 08^{\prime} \mathrm{W}$ ), alt $350 \mathrm{~m}$. Coll and subm 1979 by D Westercamp.

Gif-4780. Quarry of Morne Ponce, MPB 22

$4620 \pm 70$

Carbonized wood in pumice flow deposit $\left(14^{\circ} 45^{\prime} \mathrm{N}, 61^{\circ} 11^{\prime} \mathrm{W}\right)$, alt $5 \mathrm{~m}$. Coll and subm 1973 by D Westercamp.

\section{Gif-4775. Road from Pointe La Mare to De La Chartreuse}

$4530 \pm 110$

Carbonized wood in nuée ardente deposit $\left(14^{\circ} 47^{\prime} \mathrm{N}, 61^{\circ} 13^{\prime} \mathrm{W}\right)$, alt $5 \mathrm{~m}$. Coll and subm 1973 by D Westercamp.

Gif-4756. Rivière La Falaise, MPB 76

$4040 \pm 110$

Carbonized wood in pumice flow unit, under recent plinean deposits $\left(14^{\circ} 50^{\prime} \mathrm{N}, 61^{\circ} 07^{\prime} \mathrm{W}\right)$, alt $190 \mathrm{~m}$. Coll by L Stieltjes and subm 1977 by D Westercamp.

Gif-4757. E Basse Pointe, MPB 77 $3960 \pm 70$

Carbonized wood in pumice flow unit $\left(14^{\circ} 52^{\prime} \mathrm{N}, 61^{\circ} 07^{\prime} \mathrm{W}\right)$, alt $2 \mathrm{~m}$. Coll by L Stieltjes and subm 1977 by D Westercamp. 
Gif-4769. Rivière La Falaise, MPB 99

$3660 \pm 70$

Charcoal in pumice breccia with volcanic bombs, lying on bicolored breccia $\left(14^{\circ} 49^{\prime} \mathrm{N}, 61^{\circ} 08^{\prime} \mathrm{W}\right)$, alt $450 \mathrm{~m}$. Coll and subm 1979 by D Westercamp.

Gif-4766. Ajoupa Bouillon, MPB 96

$2300 \pm 60$

Wood in lahar $\left(14^{\circ} 49^{\prime} \mathrm{N}, 61^{\circ} 07^{\prime} \mathrm{W}\right)$, alt $210 \mathrm{~m}$. Coll and subm 1979 by D Westercamp.

\section{Gif-4754. Road from Morne Capot to Fond Labour,} MPB 74

$2250 \pm 60$

Charcoal in fine nuée ardente deposit $\left(14^{\circ} 49^{\prime} \mathrm{N}, 61^{\circ} 06^{\prime} \mathrm{W}\right)$, alt $180 \mathrm{~m}$. Coll by L Stieltjes and subm 1977 by D Westercamp.

Gif-4762. Rivière Capot, MPB 92

$2160 \pm 90$

Wood in lahar $\left(14^{\circ} 48^{\prime} \mathrm{N}, 61^{\circ} 07^{\prime} \mathrm{W}\right)$, alt $210 \mathrm{~m}$. Coll and subm 1973 by D Westercamp.

Gif-4774. Route de la Grande Savane, MPB 8

$2020 \pm 60$

Carbonized tree trunks $\left(14^{\circ} 47^{\prime} \mathrm{N}, 61^{\circ} 13^{\prime} \mathrm{W}\right)$, alt $50 \mathrm{~m}$. Coll and subm 1973 by D Westercamp.

Gif-4781. Rivière Sèche, MPB 23

$1720 \pm 60$

Carbonized wood in pumice flow deposit overlain by recent deposits $\left(14^{\circ} 46^{\prime} \mathrm{N}, 61^{\circ} 11^{\prime} \mathrm{W}\right)$, alt $100 \mathrm{~m}$. Coll and subm 1973 by D Westercamp.

Gif-4755. Savanne Pascal, MPB 75

$1700 \pm 90$

Carbonized wood in pumice flow unit overlying nuće ardente deposit $\left(14^{\circ} 49^{\prime} \mathrm{N}, 61^{\circ} 07^{\prime} \mathrm{W}\right)$. Coll by L Stieltjes and subm 1977 by $\mathrm{D}$ Westercamp.

Gif-4773. Ravine de Fond Canonville, MPB 2

$1670 \pm 90$

Carbonized wood in pumice flow deposit, $\left(14^{\circ} 47^{\prime} \mathrm{N}, 61^{\circ} 12^{\prime} \mathrm{W}\right)$, alt $150 \mathrm{~m}$. Coll and subm 1973 by D Westercamp.

Gif-4779. Rivière du Pêcheur, MPB 21

$640 \pm 60$

Carbonized tree trunks in coarse breccia $\left(14^{\circ} 48^{\prime} \mathrm{N}, 61^{\circ} 13^{\prime} \mathrm{W}\right)$, alt $40 \mathrm{~m}$. Coll and subm 1973 by D. Westercamp.

Gif-4750. Rivière Cloche, MPB 70

$620 \pm 90$

Wood in peaty clay. Coll and subm 1977 by D Westercamp.

Gif-4758. Quarry of Fond Canonville, MPB 78

$\leq \mathbf{8 0}$

Carbonized wood in pumice flow unit $\left(14^{\circ} 47^{\prime} \mathrm{N}, 61^{\circ} 12^{\prime} \mathrm{W}\right)$, alt $10 \mathrm{~m}$. Coll and subm 1977 by D Westercamp.

General Comment: these results and those obtained from Guadeloupe show some periods of strong volcanic activity common to both islands. 
Gif-2965. Montagne Pelée, MR 182

Carbonized wood in cinders, Montagne Pelée $\left(14^{\circ} 47^{\prime} \mathrm{N}, 61^{\circ} 11^{\prime} \mathrm{W}\right)$. Coll and subm 1973 by F Colmet Daage, ORSTOM, Fort de France, Martinique.

Gif-3257. Montagne Pelée, MR 190

$2820 \pm 100$

Wood in ashy breccia, 1.5 to $3 \mathrm{~m}$ thick, Montagne Pelée. Coll and subm 1973 by F Colmet Daage.

\section{La Réunion Islands}

\section{La Reunion Is. series}

Samples coll 1979 by L Stieltjes, BRGM, Saint Denis, to date end of activity of Piton des Neiges.

Gif-4865. Piton des Neiges, RU 40

$24,500 \pm 420$

Charcoal in pyroclastic deposit overlying basaltic lava flow, Piton des Neiges, Forêt de Bebour (2 $\left.1^{\circ} 07^{\prime} \mathrm{S}, 55^{\circ} 35^{\prime} \mathrm{E}\right)$.

Gif-4866. Piton des Neiges, RU 116

$\mathbf{2 6 , 7 0 0} \pm \mathbf{5 5 0}$

Paleosol in pyroclastic deposit overlying basaltic lava flow, Piton des Neiges, Forêt de Bebour (210 $\left.07^{\prime} \mathrm{S}, 55^{\circ} 35^{\prime} \mathrm{E}\right)$.

Gif-4870. Piton des Neiges, RU 167

$21,900 \pm 300$

Charcoal in pyroclastic deposit overlying basaltic lava flow, Piton des Neiges, Plateau de Belouve (21 $\left.04^{\prime} \mathrm{S}, 55^{\circ} 35^{\prime} \mathrm{E}\right)$.

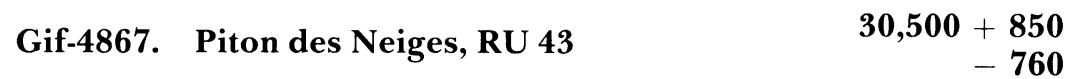

Carbonaceous matter in pyroclastic deposit lying on basaltic lava flow, Piton des Neiges, Plateau de Belouve $\left(21^{\circ} 06^{\prime} \mathrm{S}, 55^{\circ} 34^{\prime} \mathrm{E}\right)$.

Gif-4869. Piton des Neiges, RU 119

$30,700+900$ $-700$

Charcoal in pyroclastic deposit lying on lava flow, Piton des Neiges, Plateau de Belouve, dated 70,000 BP by K-Ar method (pers commun).

Gif-4868. Piton des Neiges, RU 118 $\geq 40,000$

Charcoal in pyroclastic deposit lying on lava flow, Piton des Neiges, Plateau de Belouve $\left(21^{\circ} 06^{\prime} \mathrm{S}, 55^{\circ} 34^{\prime} \mathrm{E}\right)$. Comment: possibly Gif-4867 and -4869 are slightly contaminated and there is only one pyroclastic deposit older than $40,000 \mathrm{yr}$.

Gif-4357. Piton des Neiges, RU 77-1 $\geq 35,000$

Wood under pyroclastic flow unit on W side of Cirque de Mafite, Piton des Neiges $\left(21^{\circ} 05^{\prime} \mathrm{S}, 55^{\circ} 30^{\prime} \mathrm{E}\right)$. Coll and subm 1977 by G Kieffer. 
Gif-5432. Hauts de Saint-André

Charcoal in tuff lying on andosol that overlies lava flow, Hauts de Saint-André $\left(20^{\circ} 58^{\prime} \mathrm{S}, 55^{\circ} 36^{\prime} \mathrm{E}\right)$.

Gif-5433. Hauts de Trois Bassins

$31,700 \pm 650$

Charcoal in yellow cinerite layer overlying last lava flows of Piton des Neiges, Hauts de Trois Bassins $\left(21^{\circ} 06^{\prime} \mathrm{S}, 55^{\circ} 21^{\prime} \mathrm{E}\right)$.

Gif-5559. Hauts de Saint Paul, RU 507

Charcoal in clayey unit underlying gray ash and scoria deposit, $10 \mathrm{~m}$ thick, on flank of Piton des Neiges, Hauts de Saint Paul $\left(21^{\circ} 02^{\prime} \mathrm{S}, 55^{\circ} 21^{\prime}\right.$ E).

\section{MARINE SEDIMENTS}

The following marine sediment samples come from:

1) Cores KW 31, KR 30, coll 1972 in Niger Delta, during Walda cruise aboard $R / V$ Jean Charcot and subm by J C Duplessy, CFR, CNRS, Gifsur-Yvette.

2) Cores MD 73004, MD 73025 coll 1973 in Indian Ocean during Osiris I cruise aboard $R / V$ Marion Dufresne and subm by J C Duplessy.

3) Cores CH 73139 c coll 1973 in N Atlantic Ocean during Faega I cruise of $R / V$ Jean Charcot.

4) Cores MD 77191, MD 77203 coll 1977 in N Indian Ocean during Osiris III expedition aboard $R / V$ Marion Dufresne and subm by J C Duplessy.

5) Core MG 6237 coll 1978 during Marcongo 8 expedition aboard $R / V L e$ Mizery and subm by P Giresse, Univ Perpignan.

These cores were analyzed mainly for oxygen isotopic ratios of foraminiferae (Duplessy et al, 1981), micropaleology, and sedimentology (Pastouret et al, 1978; Giresse et al, 1982). Dated levels correspond to characteristic points of isotopic curves. According to core, organic matter (OM) or carbonate fraction $\leq 44 \mu\left(\mathrm{Ca} \mathrm{CO}^{3}\right)$ has been dated. These data are presented in Table 1 .

\section{Mediterrannean Sea}

\section{Messina abyssal plain series}

Organic matter of marine sediments of cores from Messina abyssal plain. Samples subm 1974 by M Melguen, CONEXO, Plouzané, Finistère.

Gif-3397. Core KS 05, 23-25cm

$\mathbf{7 9 3 0} \pm \mathbf{5 7 0}$

Sapropelic level from core KS $05\left(38^{\circ} 35^{\prime} \mathrm{N}, 17^{\circ} 52^{\prime} \mathrm{E}\right)$, water depth $3482 \mathrm{~m}$.

Gif-3398. Core KS 05, 869-871 cm

$\geq 25,000$

Gif-3399. Core KS 06, $1315-1317 \mathrm{~cm}$

$9100 \pm 680$ $3975 \mathrm{~m}$.

Sapropelic level from core KS $06\left(36^{\circ} 69^{\prime} \mathrm{N}, 18^{\circ} 31^{\prime} \mathrm{E}\right)$, water depth 
TABLE 1

Gif Marine Sediment Samples

\begin{tabular}{|c|c|c|c|c|c|c|}
\hline $\begin{array}{l}\text { Gif } \\
\text { no. }\end{array}$ & Core no. & Lat, Long & $\begin{array}{l}\text { Water } \\
\text { depth } \\
(\mathrm{m})\end{array}$ & $\begin{array}{l}\text { Level } \\
(\mathrm{cm})\end{array}$ & Age (BP) & $\begin{array}{l}\text { Dated } \\
\text { fraction }\end{array}$ \\
\hline-4005 & $\mathrm{CH} 73139 \mathrm{c}$ & $54^{\circ} 38^{\prime} \mathrm{N}, 16^{\circ} 21^{\prime} \mathrm{W}$ & 2209 & $7-13$ & $1970 \pm 200$ & $\mathrm{Ca} \mathrm{CO}{ }^{3}$ \\
\hline-4323 & $\mathrm{CH} 73139 \mathrm{c}$ & $54^{\circ} 38^{\prime} \mathrm{N}, 16^{\circ} 21^{\prime} \mathrm{W}$ & 2209 & $46-50$ & $5170 \pm 260$ & $\mathrm{Ca} \mathrm{CO}^{3}$ \\
\hline-4006 & CH 73139 c & $54^{\circ} 38^{\prime} \mathrm{N}, 16^{\circ} 21^{\prime} \mathrm{W}$ & 2209 & $77-85$ & $8280 \pm 350$ & $\mathrm{Ca} \mathrm{CO}{ }^{3}$ \\
\hline-5150 & CH $73139 \mathrm{c}$ & $54^{\circ} 38^{\prime} \mathrm{N}, 16^{\circ} 21^{\prime} \mathrm{W}$ & 2209 & $100-102.5$ & $8960 \pm 400$ & $\mathrm{Ca} \mathrm{CO}{ }^{3}$ \\
\hline-4491 & CH 73139 c & $54^{\circ} 38^{\prime} \mathrm{N}, 16^{\circ} 21^{\prime} \mathrm{W}$ & 2209 & $112-120$ & $11,600 \pm 480$ & $\mathrm{CaCO}{ }^{3}$ \\
\hline-5152 & CH 73139 c & $54^{\circ} 38^{\prime} \mathrm{N}, 16^{\circ} 21^{\prime} \mathrm{W}$ & 2209 & $135-137$ & $11,680 \pm 540$ & $\mathrm{Ca} \mathrm{CO}^{3}$ \\
\hline--4252 & $\mathrm{CH} 73139 \mathrm{c}$ & $54^{\circ} 38^{\prime} \mathrm{N}, 16^{\circ} 21^{\prime} \mathrm{W}$ & 2209 & $140-144$ & $11,490 \pm 480$ & $\mathrm{CaCO}$ \\
\hline-5135 & $\mathrm{CH} 73139 \mathrm{c}$ & $54^{\circ} 38^{\prime} \mathrm{N}, 16^{\circ} 21^{\prime} \mathrm{W}$ & 2209 & $145-147$ & $12,880 \pm 700$ & $\mathrm{CaCO}{ }^{3}$ \\
\hline-5169 & $\mathrm{CH} 73139 \mathrm{c}$ & $54^{\circ} 38^{\prime} \mathrm{N}, 16^{\circ} 21^{\prime} \mathrm{W}$ & 2209 & $152-155$ & $13,600 \pm 670$ & $\mathrm{Ca} \mathrm{CO}{ }^{3}$ \\
\hline-4380 & $\mathrm{CH} 73139 \mathrm{c}$ & $54^{\circ} 38^{\prime} \mathrm{N}, 16^{\circ} 21^{\prime} \mathrm{W}$ & 2209 & $157.5-160.5$ & $14,800 \pm 770$ & $\mathrm{Ca} \mathrm{CO}^{3}$ \\
\hline-4253 & CH $73139 \mathrm{c}$ & $54^{\circ} 38^{\prime} \mathrm{N}, 16^{\circ} 21^{\prime} \mathrm{W}$ & 2209 & $180-184$ & $16,480=900$ & $\mathrm{Ca} \mathrm{CO}^{3}$ \\
\hline-4254 & CH $73139 c$ & $54^{\circ} 38^{\prime} \mathrm{N}, 16^{\circ} 21^{\prime} \mathrm{W}$ & 2209 & $220-224$ & $22,630 \pm 2100$ & $\mathrm{Ca} \mathrm{CO}{ }^{3}$ \\
\hline-3950 & KW 31 & $03^{\circ} 31^{\prime} \mathrm{N}, 05^{\circ} 34^{\prime} \mathrm{W}$ & 1181 & $0-6$ & $2660 \pm 250$ & $\mathrm{OM}$ \\
\hline-3951 & KW 31 & $03^{\circ} 31^{\prime} \mathrm{N}, 05^{\circ} 34^{\prime} \mathrm{W}$ & 1181 & $27-35$ & $3670 \pm 300$ & OM \\
\hline-3949 & KW 31 & $03^{\circ} 31^{\prime} \mathrm{N}, 05^{\circ} 34^{\prime} \mathrm{W}$ & 1181 & $120-125$ & $6750 \pm 410$ & $\mathrm{OM}$ \\
\hline-3959 & KW 31 & $03^{\circ} 31^{\prime} \mathrm{N}, 05^{\circ} 34^{\prime} \mathrm{W}$ & 1181 & $312-316$ & $10,900 \pm 650$ & OM \\
\hline-3813 & KW 31 & $03^{\circ} 31^{\prime} \mathrm{N}, 05^{\circ} 34^{\prime} \mathrm{W}$ & 1181 & $397-400$ & $11,200 \pm 650$ & $\mathrm{OM}$ \\
\hline-3752 & KW 31 & $03^{\circ} 31^{\prime} \mathrm{N}, 05^{\circ} 34^{\prime} \mathrm{W}$ & 1181 & $705-708$ & $11,500 \pm 650$ & $\mathrm{OM}$ \\
\hline-3815 & KW 31 & $03^{\circ} 31^{\prime} \mathrm{N}, 05^{\circ} 34^{\prime} \mathrm{W}$ & 1181 & $817-820$ & $13,600 \pm 600$ & $\mathrm{OM}$ \\
\hline-3816 & KW 31 & $03^{\circ} 31^{\prime} \mathrm{N}, 05^{\circ} 34^{\prime} \mathrm{W}$ & 1181 & $877-880$ & 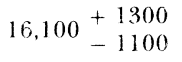 & $\mathrm{OM}$ \\
\hline-3930 & KW 31 & $03^{\circ} 31^{\prime} \mathrm{N}, 05^{\circ} 34^{\prime} \mathrm{W}$ & 1181 & $1192-1195$ & $\begin{array}{r}24,600+2400 \\
-1800\end{array}$ & $\mathrm{OM}$ \\
\hline-4106 & KW 31 & $03^{\circ} 31^{\prime} \mathrm{N}, 05^{\circ} 34^{\prime} \mathrm{W}$ & 1181 & $1297-1300$ & $\geq 24,900$ & $\mathrm{OM}$ \\
\hline-3952 & KW 31 & $03^{\circ} 31^{\prime} \mathrm{N}, 05^{\circ} 34^{\prime} \mathrm{W}$ & 1181 & $1407-1410$ & $\geq 26,000$ & $\mathrm{OM}$ \\
\hline-3957 & KR 30 & $03^{\circ} 31^{\prime} \mathrm{N}, 05^{\circ} 34^{\prime} \mathrm{W}$ & 1181 & $3-8$ & $2950 \pm 250$ & $O M$ \\
\hline-3309 & MD 73004 & $04^{\circ} 58^{\prime} \mathrm{S}, 61^{\circ} 40^{\prime} \mathrm{E}$ & 3930 & $35-40$ & $8300 \pm 350$ & $\mathrm{Ca} \mathrm{CO}^{3}$ \\
\hline-3310 & MD 73004 & $04^{\circ} 58^{\prime} \mathrm{S}, 61^{\circ} 40^{\prime} \mathrm{E}$ & 3930 & $65-70$ & $13,500 \pm 600$ & $\mathrm{CaCO}{ }^{3}$ \\
\hline-4534 & MG 237 & $5^{\circ} 12^{\prime} \mathrm{S}, 11^{\circ} 20^{\prime} \mathrm{E}$ & 1000 & $140-150$ & $8380 \pm 150$ & $\mathrm{OM}$ \\
\hline-5197 & MG 237 & $5^{\circ} 12^{\prime} \mathrm{S}, 11^{\circ} 20^{\prime} \mathrm{E}$ & 1000 & $210-220$ & $10,350 \pm 300$ & $\mathrm{OM}$ \\
\hline-4535 & MG 237 & $5^{\circ} 12^{\prime} \mathrm{S}, 11^{\circ} 20^{\prime} \mathrm{E}$ & 1009 & $290-300$ & $10,290 \pm 180$ & $\mathrm{OM}$ \\
\hline-4456 & MG 237 & $5^{\circ} 12^{\prime} \mathrm{S}, 11^{\circ} 20^{\prime} \mathrm{E}$ & 1004 & $350-360$ & $11,230 \pm 200$ & OM \\
\hline-5198 & MG 237 & $5^{\circ} 12^{\prime} \mathrm{S}, 11^{\circ} 20^{\prime} \mathrm{E}$ & 1000 & $390-400$ & $12,690 \pm 300$ & $\mathrm{OM}$ \\
\hline-4457 & MG 237 & $5^{\circ} 12^{\prime} \mathrm{S}, 11^{\circ} 20^{\prime} \mathrm{E}$ & 1000 & $470-480$ & $13.870 \pm 250$ & OM \\
\hline-4536 & MG 237 & $5^{\circ} 12^{\prime} \mathrm{S}, 11^{\circ} 20^{\prime} \mathrm{E}$ & 1000 & $590-600$ & $15,850 \pm 410$ & $\mathrm{OM}$ \\
\hline-4434 & MD 73025 & $43^{\circ} 49^{\prime} \mathrm{S}, 51^{\circ} 19^{\prime} \mathrm{E}$ & 3284 & $35-39$ & $6690 \pm 330$ & $\mathrm{Ca} \mathrm{CO}^{3}$ \\
\hline-4435 & MD 73025 & $43^{\circ} 49^{\prime} \mathrm{S}, 51^{\circ} 19^{\prime} \mathrm{E}$ & 3284 & $76-80$ & $8000 \pm 370$ & $\mathrm{Ca} \mathrm{CO}^{3}$ \\
\hline-4436 & MD 73025 & $43^{\circ} 49^{\prime} \mathrm{S}, 51^{\circ} 19^{\prime} \mathrm{E}$ & 3284 & $152-159$ & $8700 \pm 400$ & $\mathrm{Ca} \mathrm{CO}{ }^{3}$ \\
\hline-4430 & MD 73025 & $43^{\circ} 49^{\prime}$ S, $51^{\circ} 19^{\prime} \mathrm{E}$ & 3284 & $182-186$ & $9650 \pm 440$ & $\mathrm{CaCO}{ }^{8}$ \\
\hline-4431 & MD 73025 & $43^{\circ} 49^{\prime} \mathrm{S}, 51^{\circ} 19^{\prime} \mathrm{E}$ & 9284 & $256 \cdots 260$ & $11,190 \pm 520$ & $\mathrm{Ca} \mathrm{CO}^{3}$ \\
\hline-4329 & MD 73025 & $43^{\circ} 49^{\prime} \mathrm{S}, 51^{\circ} 19^{\prime} \mathrm{E}$ & 3284 & $276-280$ & $12,620 \pm 550$ & $\mathrm{Ca} \mathrm{CO}^{3}$ \\
\hline-4437 & MD 73025 & $43^{\circ} 49^{\prime} \mathrm{S}, 51^{\circ} 19^{\prime} \mathrm{E}$ & 3284 & $324-329$ & $17.200 \pm 1000$ & $\mathrm{Ca} \mathrm{CO}^{3}$ \\
\hline-4438 & MD 73025 & $43^{\circ} 49^{\prime} \mathrm{S}, 51^{\circ} 19^{\prime} \mathrm{F}$ & 3284 & $355-359$ & $19,000 \pm 1200$ & $\mathrm{Ca} \mathrm{CO}^{3}$ \\
\hline-4439 & MD 73025 & $43^{\circ} 49^{\prime} \mathrm{S}, 51^{\circ} 19^{\prime} \mathbf{E}$ & 3284 & $416-420$ & $24,000 \pm 2400$ & $\mathrm{Ca} \mathrm{CO}^{3}$ \\
\hline-4935 & MD 77191 & $7^{\circ} 30^{\prime} \mathrm{N}, 76^{\circ} 43^{\circ} \mathrm{E}$ & 1254 & $0-2$ & $2650 \pm 230$ & $\mathrm{OM}$ \\
\hline-4891 & MD 77191 & $7^{\circ} 30^{\prime} \mathrm{N}, 76^{\circ} 43^{\circ} \mathrm{E}$ & 1254 & $58-60$ & $3300 \pm 240$ & $\mathrm{OM}$ \\
\hline-4892 & MD 77191 & $7^{\circ} 30^{\prime} \mathrm{N}, 76^{\circ} 43^{\circ} \mathrm{E}$ & 1254 & $144-146$ & $3500 \pm 250$ & $\mathrm{OM}$ \\
\hline-4893 & MD 77191 & $7^{\circ} 30^{\prime} \mathrm{N}, 76^{\circ} 43^{\circ} \mathrm{E}$ & 1254 & $254-257$ & $5400 \pm 290$ & $\mathrm{OM}$ \\
\hline-4894 & MD 77191 & $7^{\circ} 30^{\prime} \mathrm{N} .76^{\circ} 43^{\circ} \mathrm{E}$ & 1254 & $403-405$ & $8160 \pm 380$ & $\mathrm{OM}$ \\
\hline-4895 & MD 77191 & $7^{\circ} 30^{\prime} \mathrm{N}, 76^{\circ} 43^{\circ} \mathrm{E}$ & 1254 & $475-478$ & $9560 \pm 440$ & $\mathrm{OM}$ \\
\hline-4896 & MD 77191 & $7^{\circ} 30^{\prime} \mathrm{N}, 76^{\circ} 43^{\circ} \mathrm{E}$ & 1254 & $502-505$ & $10,580 \pm 480$ & $\mathrm{OM}$ \\
\hline-4945 & MD 77191 & $7^{\circ} 30^{\prime} \mathrm{N}, 76^{\circ} 43^{\circ} \mathrm{E}$ & 1254 & $536-540$ & $11,370 \pm 520$ & OM \\
\hline-4937 & MD 77191 & $7^{\circ} 30^{\prime} \mathrm{N}, 76^{\circ} 43^{\circ} \mathrm{E}$ & 1254 & $596-600$ & $12,130 \pm 570$ &. $\mathrm{OM}$ \\
\hline-4946 & MD 77191 & $7^{\circ} 30^{\prime} \mathrm{N}, 76^{\circ} 43^{\circ} \mathrm{E}$ & 1254 & $641-645$ & $13,800 \pm 680$ & $\mathrm{OM}$ \\
\hline-4899 & MD 77191 & $7^{\circ} 30^{\prime} \mathrm{N}, 76^{\circ} 43^{\circ} \mathrm{E}$ & 1254 & $702-705$ & $18,100 \pm 500$ & OM \\
\hline-5754 & MD 77203 & $20^{\circ} 41^{\prime} \mathrm{N}, 59^{\circ} 34^{\prime} \mathrm{E}$ & 2442 & $15-19$ & $7920 \pm 370$ & $\mathrm{Ca} \mathrm{CO}^{3}$ \\
\hline-5755 & MD 77203 & $20^{\circ} 41^{\prime} \mathrm{N}, 59^{\circ} 34^{\prime} \mathrm{E}$ & 2442 & $77-79$ & $11,070 \pm 370$ & $\mathrm{Ca} \mathrm{CO}{ }^{3}$ \\
\hline-5756 & MD 77203 & $20^{\circ} 41^{\prime} \mathrm{N}, 59^{\circ} 34^{\prime} \mathrm{E}$ & 2442 & $143-145$ & $14,800 \pm 260$ & $\mathrm{Ca} \mathrm{CO}^{3}$ \\
\hline-5858 & MD 77203 & $20^{\circ} 41^{\prime} \mathrm{N}, 59^{\circ} 34^{\prime} \mathrm{E}$ & 2442 & $204-206$ & $17,050 \pm 400$ & $\mathrm{Ca} \mathrm{CO}^{3}$ \\
\hline-5757 & MD 77203 & $20^{\circ} 41^{\prime} \mathrm{N}, 59^{\circ} 34^{\prime} \mathrm{E}$ & 2442 & $243-245$ & $22,400 \pm 500$ & $\mathrm{Ca} \mathrm{CO}^{3}$ \\
\hline-5758 & MD 77203 & $20^{\circ} 4 \mathrm{i}^{\prime} \mathrm{N}, 59^{\circ} 34^{\prime} \mathrm{E}$ & 2442 & $313-315$ & $24,500 \pm 270$ & $\mathrm{Ca} \mathrm{CO}^{3}$ \\
\hline-5759 & MD 77203 & $20^{\circ} 41^{\prime} \mathrm{N}, 59^{\circ} 34^{\prime} \mathrm{E}$ & 2442 & $348-350$ & $25,500 \pm 450$ & $\mathrm{Ca} \mathrm{CO}^{3}$ \\
\hline-5760 & MD 77203 & $20^{\circ} 41^{\prime} \mathrm{N}, 59^{\circ} 34^{\prime} \mathrm{E}$ & 2442 & $400-403$ & $26,800 \pm 1000$ & $\mathrm{Ca} \mathrm{CO}^{3}$ \\
\hline-5860 & MD 77203 & $20^{\circ} 41^{\prime} \mathrm{N}, 59^{\circ} 34^{\prime} \mathrm{E}$ & 2442 & $503-506$ & $29,200 \pm 1000$ & $\mathrm{Ca} \mathrm{CO}^{3}$ \\
\hline-5861 & MD 77203 & $20^{\circ} 41^{\prime} \mathrm{N}, 59^{\circ} 34^{\prime} \mathrm{E}$ & 2442 & $603-605$ & $31,200 \pm 900$ & $\mathrm{Ca} \mathrm{CO}^{3}$ \\
\hline
\end{tabular}


Gif-3400. Core KS 07, 31-33cm

$8700 \pm 680$ $3915 \mathrm{~m}$

Sapropelic level from core $\mathrm{KS} 07\left(35^{\circ} 40^{\prime} \mathrm{N}, 17^{\circ} 13^{\prime} \mathrm{E}\right)$, water depth

Gif-3401. Core KS 07, 39-40cm

$9600 \pm 1200$

Sapropelic level.

\section{Venezuela}

\section{Dmerara plain series}

Organic fraction of marine sediments cores coll 1973 during Orgon II cruise on board $R / V$ Jean Charcot. Subm 1978 by J Moyes, Univ Bordeaux.

\section{Gif-4543. Core KS 02}

$9600 \pm 440$

Carbonate fraction $(\geq 60 \mu)$ from level 92.5 to $97.5 \mathrm{~cm}$ of core KS 02 $\left(10^{\circ} 28^{\prime} \mathrm{N}, 59^{\circ} 30^{\prime} 59^{\circ} 30^{\prime} \mathrm{W}\right)$, water depth $1260 \mathrm{~m}$. Comment: sample dated because of abundance of Pteropods in sediment at this level.

Gif-4544. Core KS 03

Carbonate fraction $(\geq 60 \mu)$ from level 380 to $390 \mathrm{~cm}$ of core KS $03\left(10^{\circ}\right.$ $\left.02^{\prime} \mathrm{N}, 57^{\circ} 32^{\prime} \mathrm{W}\right)$, water depth $3410 \mathrm{~m}$. Comment: sample subm to date disappearance of Pulleniatina obliloquilaculata sp.

\section{RERERENCES}

Bonhommet, N and Zähringer, J, 1969, Paleomagnetism and potassium-argon determination of the Laschamp geomagnetic polarity event: EPSL, v 6, p 43.

Chollet, A, Reigner, H and Boutin, P, 1974, La Grotte du Bois-Ragot à Gouex, Vienne: Gallia Préhist, v 17, no. 1, p 285-291.

Clottes, J and Simmonet, R, 1977, Le Paléolithique final dans le bassin de Tarascon-surAriège, d'après les gisements des Eglises (Ussat) et de Rhodes II (Arignac): Colloque internatl CNRS, no. 271. La Fin des Temps Glaciaires en Europe, p 647-659.

Crisci, G M. Delibrias, G, De Rosa, R, Mazzuoli, R and Sheridan, M F, 1983, Age and petrology of the Late-Pleistocene brown tuffs on Lipari, Italy: Bull Volcanol, v 46, no. 4, p $381-391$.

Bayle des Hermens, de, R, 1977, Les niveaux supérieurs du Magdalénien de la Grotte du Rond du Barry, Polignac (Haute Loire): Colloque Internatl CNRS, La Fin des Temps Glaciaires en Europe, v 271, p 601-611.

Delibrias, G, Guillier, M T and Labeyrie J, 1970, Gif natural radiocarbon measurements: Radiocarbon, v 12, p 421-443. 319 . 94.

Delibrias G, Le Roy Ladurie, M and Le Roy Ladurie, E, 1975, La forêt fossile de Gründelwald: nouvelles datations: Ann Econ Soc Civilisations, v 1, p 137-145.

Delibrias, G, Di Paola, G M, Rosi, M and Santacroce, R, 1979. La storia errutiva del complesso vulcanico Somma Vesuvio ricostruito dalle successioni piroclastiche del Monte Somma: Rendiconti Soc Ital Min Petrol, v 35, no. 1, p 411-438.

Delporte, H, 1984, Le grand abri de la Ferrassie: Etudes quaternaires, v 7, Univ Provence, $277 \mathrm{p}$.

Descamps, C, Thilmans, G and Thommeret, Y, 1974, Données sur l'édification de l'amas coquillier de Dioron Boumak, Sénégal, Ass Seneg, Et Quatern Af: Bull liaison, Senegal, v 41, p $67-83$.

Duplessy, J C, Delibrias, G, Turon, J L, Pujol, C and Duprat, J, 1981, Deglacial warming of the Northeastern Atlantic ocean: Correlation with the paleoclimatic evolution of the European continent: Paleogeog, Paleoclimatol, Paleoecol, v 35, p 121-144.

Evin, J, Maréchal, J and Marien, G, 1983, Lyon natural radiocarbon measurements IX: Radiocarbon, v 25, p 59-128. 
Gagnière, S, de Lanfranchi, F, Miskovsky, J C, Prost, M, Renault-Miskovski, J and Weiss, M C, 1969, L'abri d'Araguina-Sennola à Bonifacio, Corse: Soc Préhist Fr Bull, v 66, p 385418 .

Gillot, P Y, Labeyrie, J, Laj, C, Valladas, G, Guérin, G, Poupeau, G and Delibrias, G, 1979: Age of Laschamp paleomagnetic excursion revised: EPSL, v 42, p 444-450.

Giresse, P, Bongo-Passi, G, Delibrias, G, and Duplessy, J C, 1982, La lithostatigraphie des sédiments hémipélagiques du delta profond du fleuve Congo et ses indications sur les paléoclimats de la fin du Quaternaire: Bull Soc Geol France, v 24, no. 7, p 813-815.

Gomez, J, 1975, Le rempart artenacien du Camp de Pierre-Dure à Voeuil-et-Gignet (Charente): Bull Soc Prehist Fr, v 72, no. 4, p $117-124$

Jalut, G, 1974, Evolution de la végétation et variations climatiques durant les quinze derniers millénaires dans l'extrémité orientale des Pyrénées: Thesis, Univ Toulouse III.

1981, Action de l'homme sur la forêt montagnarde des Pyrénées ariégeoises et orientales, depuis 4000 BP d'après l'analyse pollinique: Act Cong Natl Soc Savantes, 106 th, Perpignan.

Kieffer, G. 1979, Activité de l'Etna pendant les derniers 20.000 ans: Acad Sci [Paris] Comptes rendus, v 282, p 1023-1026.

1985, Evolution structurale et dynamique d'un grand volcan polygénique: stade d'édification et d'activité actuelle de l'Etna (Sicile): Thesis, Univ Clermont-Ferrand II.

Klein, J, Lerman, J C, Damon, P E and Ralph, E K, 1982, Calibration of radiocarbon dates: tables based on the consensus data of the Workshop on Calibrating the Radiocarbon Time Scale: Radiocarbon, v 24, p 103-150.

Laming-Emperaire, A, 1979, Missions archéologiques franco-brésiliennes de Lagoa Santa Minas Gerais, Brésil. Le Grand abri de Lapa Vermelha (PL) Revista de Pré-Historia, Sao Paulo, I (I) p 53-89.

Laugénie, C, 1982, La région des lacs, Chili Méridional: Thesis doc, Univ Bordeaux III.

Longin, R, 1971, New method of collagen extraction for radiocarbon dating: Nature, $v 230, \mathrm{p}$ $241-242$.

Markgraf, V, 1977, New dates on the late and Postglacial Vegetational History of "La Mission," Tierra del Fuego, Argentina: IV Intern Palyno Conf Lucknow, India, 1-12.

Pastouret, L, Chamley, H, Delibrias, G and Duplessy, J C, 1978, Late Quaternary climatic changes in Western Tropical Africa deduced from deep-sea sedimentation off the Niger delta: Oceanologica Acta, 1, 2, p 217-232.

Paterne, M, 1980, Chronologie des eruptions récentes du Massif de la Soufrière (Guadeloupe, Petites Antilles). Essai de comparaison des périodes d'activité volcanique de quelques grandes régions volcaniques. Thesis of 3 ème cycle, Univ Bordeaux I.

Pétrequin, P, Urlacher, J P, and Vuaillat, D, 1969, Habitat et sépultures de l'âge du Bronze final à Dampierre-sur-le-Doubs (Doubs): Gallia Préhist, XII, v 1, p 1-35.

Simmonet, R, 1967, L'abri sous roche Rhodes II et la question de l'Azilien dans les Pyrénées Fraçaises: Soc Préhist France Bull, 64, v 1, p 175-186.

Tancredi, A, Sieffermann, G, Besnus, Y, Fusil, G and Delibrias, G, 1975, Présence et Formation de niveaux de sidérite dans les sédiments récents du delta amazonien: Bull Groupe franç Argiles, t XXVII, p 13-29.

Veyret, Y, Brousse, R and Delibrias, G. 1978, Première datation d'un épisode glaciaire récent dans le Massif Central français: Acad Sci [Paris] Comptes rendus, v 286, p 1089-1092. 